\title{
Existence of solutions to a two-dimensional model for nonisothermal two-phase flows of incompressible fluids
}

\author{
Michela Eleuteri ${ }^{\mathrm{a}, 1}$, Elisabetta Rocca ${ }^{\mathrm{b}, \mathrm{c}, 2}$, Giulio Schimperna ${ }^{\mathrm{d}, *, 3}$ \\ a Dipartimento di Matematica ed Informatica "U. Dini”, viale Morgagni 67/a, I-50134 Firenze, Italy \\ b Weierstrass Institute for Applied Analysis and Stochastics, Mohrenstrasse 39, D-10117 Berlin, Germany \\ c Dipartimento di Matematica "F. Enriques", Università degli Studi di Milano, I-20133 Milano, Italy \\ d Dipartimento di Matematica "F. Casorati”, Università degli Studi di Pavia, via Ferrata 1, I-27100 Pavia, Italy
}

Received 6 June 2014; received in revised form 28 April 2015; accepted 31 May 2015

Available online 23 June 2015

\begin{abstract}
We consider a thermodynamically consistent diffuse interface model describing two-phase flows of incompressible fluids in a non-isothermal setting. The model was recently introduced in [11] where existence of weak solutions was proved in three space dimensions. Here, we aim to study the properties of solutions in the two-dimensional case. In particular, we can show existence of global in time solutions satisfying a stronger formulation of the model with respect to the one considered in [11]. (C) 2015 L'Association Publications de l'Institut Henri Poincaré. Published by Elsevier B.V. All rights reserved.
\end{abstract}

MSC: 35Q35; 35K25; 76D05; 35D30

Keywords: Cahn-Hilliard; Navier-Stokes; Incompressible non-isothermal binary fluid; Global-in-time existence; A-priori estimates

\section{Introduction}

We consider here a mathematical model for two-phase flows of non-isothermal incompressible fluids in a bounded container $\Omega$ in $\mathbb{R}^{2}$ during a finite time interval $(0, T)$, with no restrictions on the magnitude of the final time $T>0$. The model consists of a PDE system describing the evolution of the unknown variables $\boldsymbol{u}$ (macroscopic velocity), $\varphi$ (order parameter), $\mu$ (chemical potential), $\vartheta$ (absolute temperature), and it takes the form

\footnotetext{
* Corresponding author.

E-mail addresses: eleuteri@math.unifi.it (M. Eleuteri), rocca@wias-berlin.de, elisabetta.rocca@unimi.it (E. Rocca), giusch04@unipv.it (G. Schimperna).

1 The author is partially supported by the FP7-IDEAS-ERC-StG Grant \#256872 (EntroPhase) and by GNAMPA (Gruppo Nazionale per l'Analisi Matematica, la Probabilità e le loro Applicazioni) of INdAM (Istituto Nazionale di Alta Matematica).

2 The author is supported by the FP7-IDEAS-ERC-StG Grant \#256872 (EntroPhase), by the GNAMPA (Gruppo Nazionale per l'Analisi Matematica, la Probabilità e le loro Applicazioni) of INdAM (Istituto Nazionale di Alta Matematica) and by the IMATI - C.N.R. Pavia.

3 The author is partially supported by the FP7-IDEAS-ERC-StG Grant \#256872 (EntroPhase) and by GNAMPA (Gruppo Nazionale per l'Analisi Matematica, la Probabilità e le loro Applicazioni) of INdAM (Istituto Nazionale di Alta Matematica).
} 


$$
\begin{aligned}
& \operatorname{div} \boldsymbol{u}=0, \\
& \boldsymbol{u}_{t}+\boldsymbol{u} \cdot \nabla \boldsymbol{u}+\nabla p=\Delta \boldsymbol{u}-\operatorname{div}(\nabla \varphi \otimes \nabla \varphi), \\
& \varphi_{t}+\boldsymbol{u} \cdot \nabla \varphi=\Delta \mu, \\
& \mu=-\Delta \varphi+F^{\prime}(\varphi)-\vartheta, \\
& \vartheta_{t}+\boldsymbol{u} \cdot \nabla \vartheta+\vartheta\left(\varphi_{t}+\boldsymbol{u} \cdot \nabla \varphi\right)-\operatorname{div}(\kappa(\vartheta) \nabla \vartheta)=|\nabla \boldsymbol{u}|^{2}+|\nabla \mu|^{2} .
\end{aligned}
$$

Relation (1.2), with the incompressibility constraint (1.1), represents a variant of the Navier-Stokes system; (1.3)-(1.4) correspond to a form of the Cahn-Hilliard system [10] for phase separation; (1.5) is the internal energy equation describing the evolution of temperature. Note that transport effects are admitted for all variables in view of the occurrence of material derivatives in (1.2), (1.3), and (1.5). As usual, the variable $p$ in the Navier-Stokes system (1.2) represents the (unknown) pressure. The function $F$ whose derivative appears in (1.4) is a possibly nonconvex potential whose minima represent the least energy configurations of the phase variable. Here we will assume that $F$ is smooth and has at most a power-like growth at infinity. Indeed, it is not clear whether our result could be extended to other classes of physically significant potential, having nonsmooth or singular character (like, e.g., the so-called logarithmic potential $F(r)=(1+r) \log (1+r)+(1-r) \log (1-r)-r^{2}$ which typically appears in CahnHilliard-based models, see, e.g., [30]). Finally, the coefficient $\kappa(\vartheta)$ in (1.5) stands for the heat conductivity of the fluid. Here we shall assume that $\kappa$ grows at infinity like a sufficiently high power of $\vartheta$ (see (2.11) below).

The PDE system (1.1)-(1.4), in the case of a constant temperature $\vartheta$, is referred to in the literature as "Model H". Even if many authors considered the isothermal Model $\mathrm{H}$ (cf., for instance, [3,19-23,25] for the physical derivation of the model, and $[1,2,4,5,15-17,27,34,41,42]$ for the study of the resulting evolution system), up to our knowledge no contributions are so far present in the literature in the non-isothermal case, except for [35], where a linearization of (1.5) is considered, [18], where the numerical analysis of a phase-field model for binary quasi-incompressible fluid with thermocapillarity effects is performed, [40], where a Cahn-Hilliard-Boussinesq system has been studied, and our previous paper [11], where system (1.1)-(1.5) has been analyzed in the 3D case.

Actually, the above model was proposed in our recent work [11] starting from the balance laws for internal energy and entropy; in particular, thermodynamical consistence was shown to hold for any (positive) value of the absolute temperature $\vartheta$. Moreover, existence of solutions for a weak formulation of (1.1)-(1.5) was proved when the system is settled in a smooth bounded domain $\Omega \subset \mathbb{R}^{3}$ and complemented with no-flux conditions for $\varphi, \mu$ and $\vartheta$ and with slip conditions for $\boldsymbol{u}$. Mathematically, the main source of difficulty in system (1.1)-(1.5) comes from the quadratic terms on the right hand side of (1.5). Their occurrence is physically motivated as one considers the derivation of the model in terms of the energy and entropy balances (cf. [11, Sec. 2]). Roughly speaking, one can say that these terms represent a source of thermal energy coming from the dissipation of kinetic energy due to viscosity (cf. (1.2)) and of configuration energy due to action of micro-forces (cf. (1.3)-(1.4)). This energy dissipation, as expected, happens in such a way to increase the entropy of the system.

From the analytical viewpoint, the quadratic terms in (1.5) can be controlled only in the $L^{1}$-norm, at least in the 3D-case. This is the reason why in [11] we introduced a suitable weak formulation along the lines of an idea originally developed in [8,12] for dealing with heat conduction in fluids, in [13] for solid-liquid phase transitions, and more recently in [32] for damage phenomena. In such a setting, the "heat" equation (1.5) is replaced with a relation describing the balance of total energy (i.e., not only of thermal energy), which does no longer contain quadratic terms. This is complemented with a distributional version of the entropy inequality.

Looking at the 2D model, whose analysis is the aim of this paper, it is well-known that, for the Navier-Stokes system (1.2), additional regularity is available provided that the forcing term (here given by $-\operatorname{div}(\nabla \varphi \otimes \nabla \varphi))$ lies in $L^{2}$ (cf., e.g., [31]). Fortunately, this seems to happen in our case, as one can readily check starting from the available energy and entropy estimates; hence, there is hope to get additional summability for the quadratic term $|\nabla \boldsymbol{u}|^{2}$ in (1.5). This was the motivation which led us to investigate whether it is possible to prove existence of a solution to the original (strong) system (1.1)-(1.5) in two space dimensions.

In order to explain our ideas, we first need to introduce some basic assumptions. First of all, to avoid technical complications related with the choice of boundary data, we ask the system to be settled in the unit torus $\Omega:=[0,1] \times$ $[0,1]$ and complemented with periodic boundary conditions for all unknowns. It is worth noting that some other types of boundary conditions could be assumed. For instance, we may take, as in [11], no-flux (i.e., homogeneous Neumann) conditions for $\varphi, \mu$ and $\vartheta$ (as it is physically reasonable if one assumes the container $\Omega$ to be insulated 
from the exterior), whereas for $\boldsymbol{u}$ we may consider any conditions that could allow the transport terms to have zero spatial mean and are compatible with the existence of smooth solutions to the 2D Navier-Stokes system (cf. estimate (3.26) below). This is the case, for instance, of homogeneous Dirichlet conditions (cf., e.g., [36, Thm. 3.10, p. 314]). The adjustments required in order to address these cases should be relatively limited. On the other hand, dealing with different types of boundary conditions, especially referring to the variable $\boldsymbol{u}$, may be mathematically much more involved.

Coming to our mathematical argument and referring to Section 3 for more details, we just give here some brief explanation of the procedure we use in order to get better regularity results. Actually, once additional regularity for $\boldsymbol{u}$ has been obtained, further estimates for $\varphi$ and $\mu$ may be obtained by differentiating in time (1.4), and testing the result by $\varphi_{t}$. This procedure, however, requires to control the product term $\vartheta_{t} \varphi_{t}$. In view of the highly nonlinear structure of (1.5), getting a bound for $\vartheta_{t}$ directly seems difficult. Hence, the only possibility seems that of testing (1.5) by $\varphi_{t}$ in order to cancel the bad term. As a drawback, we have to provide some bound for the quadratic terms on the right hand side. In particular, the crucial point stands in the control of $|\nabla \mu|^{2}$, for which only an $L^{1}$-estimate is available at this level. Actually, this term is estimated by means of a sharp two-dimensional interpolation-embedding inequality which is proved in Appendix A. This inequality, indeed, follows from well-known two-dimensional embedding theorems; however we were not able to find any specific reference. The underlying idea, which is not at all new but which we prefer to reproduce here for the reader's convenience, stands in optimizing with respect to $q$ the embedding constant of the immersion $\|v\|_{H^{1}(\Omega)^{\prime}} \leq c_{q}\|v\|_{L^{q}(\Omega)}$ which holds true in 2D for every $q \in(1,+\infty]$ and $v \in L^{q}(\Omega)$ (cf. [37, (17), p. 479]). Alternatively, one may deduce the inequality more directly from the Moser-Trudinger embedding inequality applying some tools from convex analysis.

With the inequality at disposal, and performing some amount of technical work, we can obtain the desired enhanced a-priori bounds. These estimates permit us to pass to the limit in a suitable approximation scheme (which is just sketched, for brevity), obtaining in this way a solution to the original (strong) system (1.1)-(1.5), in a proper regularity class, coupled with periodic boundary conditions and with the initial conditions.

It is finally worth noting that, while in [11] we needed to assume non-constant specific heat and heat conductivity, both having a suitable growth at 0 and at $\infty$ (the reasons were mainly of mathematical type; namely, we needed to get a sufficient summability for $\vartheta$ ), here we just need a sufficiently fast growing heat conductivity (cf. [39] for a physical justification of this choice), but we can allow for a constant specific heat. Actually, we have to notice that, if from one side this choice is more mathematically challenging because it gives less summability in $\vartheta$, however, it is also mandatory for our analysis, since it is needed in order to perform the regularity estimates on the combined Cahn-Hilliard-temperature system (cf. Remark 3.1 below). For the same reason (i.e., to get more regularity on the velocity field $\boldsymbol{u}$ ), we need to assume a constant viscosity $v$ (equal to 1 for simplicity). This is however quite standard in the framework of Navier-Stokes equations.

The arguments given in this paper may also be adapted to deal with other interesting related models. For instance, we could consider the case when the "Cahn-Hilliard" relations (1.3)-(1.4) are replaced by their "Allen-Cahn" equivalent (cf. [9])

$$
\varphi_{t}+\boldsymbol{u} \cdot \nabla \varphi-\Delta \varphi+F^{\prime}(\varphi)-\vartheta=0 .
$$

Moreover, as a byproduct of our results, one can deduce the existence of solutions in 2D for the so-called Frémond's model of phase transitions with microscopic effects introduced in [14], at least in the case of power-like heat conductivity. The Frémond model basically corresponds to system (1.1)-(1.5) where the velocity $\boldsymbol{u}$ is assumed to be identically equal to 0 . Indeed, for this model, in the case of Neumann boundary conditions and standard Fourier heat flux law, existence of global in time "strong" solutions was known only in the one-dimensional setting (cf. [28,29]), while weak solutions were proved to exist in 3D (cf. [13]) when (1.5) is replaced by the total energy balance and an entropy inequality. Hence, this paper covers the missing 2D case, at least for the case of power-like heat conductivity and periodic boundary conditions.

Let us finally note that uniqueness of solutions, as well as their long-time behavior (both in terms of trajectories and of attractors) for the whole system (1.1)-(1.5), are still open issues. Actually, uniqueness of solution to the Model $\mathrm{H}$ corresponding to the isothermal version of our system has been proved in [1] and [4]. Extending these results to the non-isothermal case, however, seems to be quite hard since the difficulty here comes mainly from the highly nonlinear temperature equation. Indeed, even neglecting the contribution of the velocity $\boldsymbol{u}$, i.e., reducing our system 
to the so-called Frémond phase-field model [14], uniqueness seems to be known only in special cases, like for instance in 1D (cf., e.g., [28] and [13]).

Here is the plan of the paper: in the next Section 2 we specify our assumptions on coefficients and data and state the precise mathematical formulation of our problem together with the related existence theorem. The remainder of the paper is devoted to the proof of the theorem. In particular, the core of our argument is given in Section 3, where we provide the a-priori estimates and the compactness argument necessary to pass to the limit in the approximation scheme. Indeed, in order to avoid technicalities, the estimates are obtained in a formal way leaving the details of a possible regularization in the subsequent Section 4. Finally, Appendix A contains the proof of the mentioned two-dimensional interpolation-embedding inequality.

\section{Assumptions and main results}

In order to state the precise mathematical formulation of our problem we first need to introduce some functional spaces. Recalling that $\Omega=[0,1] \times[0,1]$, we note as $H:=L_{\text {per }}^{2}(\Omega)$ the space of functions in $L^{2}\left(\mathbb{R}^{2}\right)$ which are $\Omega$-periodic (i.e., 1-periodic both in $x_{1}$ and in $x_{2}$ ). Analogously, we set $V:=H_{\text {per }}^{1}(\Omega)$. The spaces $H$ and $V$ are endowed with the norms of $L^{2}(\Omega)$ and $H^{1}(\Omega)$, respectively. For brevity, the norm in $H$ will be simply indicated by $\|\cdot\|$. We will note by $\|\cdot\|_{X}$ the norm in the generic Banach space $X$. The symbol $\langle\cdot, \cdot\rangle$ will indicate the duality between $V^{\prime}$ and $V$ and $(\cdot, \cdot)$ will stand for the scalar product of $H$. We also write $L^{p}(\Omega)$ in place of $L_{\text {per }}^{p}(\Omega)$, and the same for other spaces; indeed, no confusion should arise since periodic boundary conditions are assumed to hold for all unknowns. Still for brevity, we use the same notation for indicating vector-valued (or tensor-valued) function spaces and related norms. For instance, writing $\boldsymbol{u} \in H$, we will in fact mean $\boldsymbol{u} \in L_{\text {per }}^{2}(\Omega)^{2}$. Also the incompressibility constraint (1.1) will not be emphasized in the notation for functional spaces (hence, the notation $\boldsymbol{u} \in H$ will also implicitly subsume that $\operatorname{div} \boldsymbol{u}=0$ in the sense of distributions). These simplifications will allow us to shorten a bit some formulas.

For any function $v \in H$, we will note as

$$
v_{\Omega}:=\frac{1}{|\Omega|} \int_{\Omega} v=\int_{\Omega} v
$$

the spatial mean of $v$. Replacing the integral with a duality pairing, the same notation will be used in case $v \in V^{\prime}$. The symbols $V_{0}, H_{0}$ and $V_{0}^{\prime}$ denote the subspaces of $V, H$ and, respectively, $V^{\prime}$ containing the function(al)s having zero spatial mean. We notice that the distributional operator $(-\Delta)$ is invertible if it is seen as a mapping from $V_{0}$ to $V_{0}^{\prime}$. In the sequel we shall denote by $\mathcal{N}$ its inverse operator.

Moreover, in the following we will frequently use the following $2 \mathrm{D}$ interpolation inequalities:

$$
\begin{aligned}
& \|v\|_{L^{4}(\Omega)} \leq c\|v\|_{V}^{1 / 2}\|v\|^{1 / 2}, \\
& \|v\|_{L^{\infty}(\Omega)} \leq c\|v\|_{H^{2}(\Omega)}^{1 / 2}\|v\|^{1 / 2}, \\
& \|v\|_{L^{r}(\Omega)} \leq c\|v\|_{L^{s}(\Omega)}^{1-\alpha}\|v\|_{L^{\infty}(\Omega)}^{\alpha}, \quad \alpha=1-\frac{s}{r},
\end{aligned}
$$

holding for any sufficiently smooth function $v$ and for suitable embedding constants, all denoted by the same symbol $c>0$ for brevity.

We will also use the following nonlinear version of the Poincaré inequality

$$
\left\|v^{p / 2}\right\|_{V}^{2} \leq c_{p}\left(\|v\|_{L^{1}(\Omega)}^{p}+\left\|\nabla v^{p / 2}\right\|^{2}\right),
$$

holding for all nonnegative $v \in L^{1}(\Omega)$ such that $\nabla v^{p / 2} \in L^{2}(\Omega)$, and for all $p \in[2, \infty)$. We also note that

$$
\|v\| \leq c\|\nabla v\|^{1 / 2}\|v\|_{V^{\prime}}^{1 / 2} \text { for all } v \in V_{0} .
$$

This property can be proved by combining the standard interpolation inequality $\|v\| \leq c\|v\|_{V}^{1 / 2}\|v\|_{V^{\prime}}^{1 / 2}$ with the Poincaré-Wirtinger inequality. 
In the sequel we will frequently use the continuous embedding $V \subset L^{p}(\Omega)$, holding for all $p \in[1, \infty)$. Moreover, it is not difficult to see that (2.6) implies

$$
\|v\|_{L^{p}(\Omega)}^{2} \leq \epsilon\|\nabla v\|^{2}+c_{\epsilon}\|v\|_{V^{\prime}}^{2} \text { for all } v \in V_{0},
$$

for all (small) $\epsilon>0$ and correspondingly large $c_{\epsilon}>0$ whose value additionally depends on $p \in[1, \infty$ ).

With the above notation at disposal, we can introduce our main assumptions. First of all, we ask the configuration potential $F$ to satisfy:

$$
\begin{aligned}
& F \in C^{2}(\mathbb{R} ; \mathbb{R}), \quad \liminf _{|r| \rightarrow \infty} \frac{F(r)}{|r|}>0, \\
& F^{\prime \prime}(r) \geq-\lambda \quad \text { for some } \lambda \geq 0 \text { and all } r \in \mathbb{R}, \\
& \left|F^{\prime \prime}(r)\right| \leq c_{F}\left(1+|r|^{p_{F}}\right) \quad \text { for some } c_{F} \geq 0, p_{F} \geq 0, \quad \text { and all } r \in \mathbb{R} .
\end{aligned}
$$

In other words, we ask for $F$ to be a smooth, coercive (in view of (2.8)), $\lambda$-convex (cf. (2.9)) function, with at most polynomial growth at infinity (cf. (2.10)). These conditions may be probably relaxed (admitting, for instance, functions with exponential growth at infinity), at the price, however, of technical complications.

Next, we assume the heat conductivity to be given by

$$
\kappa(r)=1+r^{q}, \quad q \in[2, \infty), \quad r \geq 0 .
$$

Correspondingly, we define

$$
K(r):=\int_{0}^{r} \kappa(s) \mathrm{d} s=r+\frac{1}{q+1} r^{q+1}, \quad r \geq 0 .
$$

In the sequel we will often need to estimate the value $\|K(\vartheta)\|_{V}^{2}$. To this aim, we first observe that, for some $k_{q}>0$,

$$
\int_{\Omega} \kappa(\vartheta)^{2}|\nabla \vartheta|^{2}=\|\nabla K(\vartheta)\|^{2} \geq\|\nabla \vartheta\|^{2}+k_{q}\left\|\nabla \vartheta^{q+1}\right\|^{2} .
$$

Then, exploiting (2.5) with the choice $p=2$ we obtain

$$
\|K(\vartheta)\|_{V}^{2} \leq c_{q}\left(\int_{\Omega}\left(\vartheta+\vartheta^{q+1}\right)\right)^{2}+c_{q}\left(\int_{\Omega}\left|\nabla \vartheta+\nabla\left(\frac{\vartheta^{q+1}}{q+1}\right)\right|^{2}\right)=: I+I I
$$

for some $c_{q}>0$. Now, using again (2.5), this time with the choice $p=2(q+1)$, we deduce

$$
I \leq c_{q}\|\vartheta\|_{L^{1}(\Omega)}^{2}+c_{q}\left(\|\vartheta\|_{L^{1}(\Omega)}^{2(q+1)}+\left\|\nabla \vartheta^{q+1}\right\|^{2}\right) \leq c_{q}\left(1+\|\vartheta\|_{L^{1}(\Omega)}^{2(q+1)}+\left\|\nabla \vartheta^{q+1}\right\|^{2}\right) .
$$

Estimating $I I$ with the help of (2.13) we then conclude

$$
\|K(\vartheta)\|_{V}^{2} \leq c_{q}\left(1+\|\vartheta\|_{L^{1}(\Omega)}^{2(q+1)}+\int_{\Omega} \kappa^{2}(\vartheta)|\nabla \vartheta|^{2}\right) .
$$

Finally we come to our assumptions on the initial data:

$$
\begin{aligned}
& \boldsymbol{u}_{0} \in V, \quad \operatorname{div} \boldsymbol{u}_{0}=0, \\
& \varphi_{0} \in H_{\text {per }}^{3}(\Omega), \\
& \vartheta_{0} \in V, \quad \vartheta_{0} \geq \underline{\vartheta}>0 \text { a.e. in } \Omega,
\end{aligned}
$$

where $\underline{\vartheta}$ is some positive constant (actually the last condition in (2.19) could be relaxed by asking $\vartheta_{0}>0$ almost everywhere with $\log \vartheta_{0} \in L^{1}(\Omega)$ ).

With the above machinery at disposal, we can conclude this section by stating our main existence theorem, whose proof will occupy the remainder of the paper: 
Theorem 2.1. Let us assume (2.8)-(2.10), (2.11), and (2.17)-(2.19). Let also $T>0$. Then, there exists at least one strong solution to the non-isothermal model for two-phase fluid flows, namely, one quadruple $(\boldsymbol{u}, \varphi, \mu, \vartheta)$ with

$$
\begin{aligned}
& \boldsymbol{u} \in H^{1}(0, T ; H) \cap L^{\infty}(0, T ; V) \cap L^{2}\left(0, T ; H^{2}(\Omega)\right), \\
& \varphi \in W^{1, \infty}\left(0, T ; V^{\prime}\right) \cap H^{1}(0, T ; V) \cap L^{2}\left(0, T ; H^{3}(\Omega)\right), \\
& \mu \in H^{1}\left(0, T ; V^{\prime}\right) \cap L^{\infty}(0, T ; V) \cap L^{2}\left(0, T ; H^{3}(\Omega)\right), \\
& \vartheta \in H^{1}\left(0, T ; V^{\prime}\right) \cap L^{\infty}\left(0, T ; L^{q+2}(\Omega)\right) \cap L^{2}(0, T ; V), \quad \vartheta>0 \quad \text { a.e.in }(0, T) \times \Omega, \\
& K(\vartheta) \in L^{2}(0, T ; V),
\end{aligned}
$$

such that the equations of the system (1.1)-(1.4) hold in the sense of distributions as well as almost everywhere in $(0, T) \times \Omega$, while (1.5) holds, for a.e. $t \in(0, T)$, as the following relation in $V^{\prime}$ :

$$
\vartheta_{t}+\boldsymbol{u} \cdot \nabla \vartheta+\vartheta\left(\varphi_{t}+\boldsymbol{u} \cdot \nabla \varphi\right)-\Delta K(\vartheta)=|\nabla \boldsymbol{u}|^{2}+|\nabla \mu|^{2},
$$

where $\Delta$ is a weak form of the Laplace operator with periodic boundary conditions. Moreover, the quadruple $(\boldsymbol{u}, \varphi, \mu, \vartheta)$ complies with the initial condition

$$
\left.\boldsymbol{u}\right|_{t=0}=\boldsymbol{u}_{0},\left.\quad \varphi\right|_{t=0}=\varphi_{0},\left.\quad \vartheta\right|_{t=0}=\vartheta_{0},
$$

almost everywhere in $\Omega$.

\section{Global existence}

We start by deriving the a-priori estimates leading to existence of weak solutions. As noted in the Introduction, we shall work directly, though formally, on the original system (1.1)-(1.5) without referring to any approximation or regularization. Indeed, this permits us to make the argument more readable and to avoid technical complications. The details of a possible regularization scheme compatible with the a priori estimates are postponed to Section 4 below. In the following, the letter $c$ will denote a generic positive constant depending only on the data of the problem, whose value is allowed to vary on occurrence. In particular, $c$ is intended to be independent of all regularization parameters.

Energy estimate. This basic property corresponds to the energy conservation principle. To deduce it, we test (1.2) by $\boldsymbol{u},(1.3)$ by $\mu,(1.4)$ by $-\varphi_{t},(1.5)$ by 1 , integrate over $\Omega$, and sum all the obtained relations together. Then, using

$$
(\boldsymbol{u} \cdot \nabla \varphi) \mu=(\boldsymbol{u} \cdot \nabla \varphi)\left(-\Delta \varphi+F^{\prime}(\varphi)-\vartheta\right)
$$

(cf. (1.4)) and performing standard integration by parts (cf. [11, Sec. 2] for more details), we infer that

$$
\frac{\mathrm{d}}{\mathrm{d} t} \mathcal{E}(\boldsymbol{u}, \varphi, \vartheta)=0, \quad \text { where } \mathcal{E}(\boldsymbol{u}, \varphi, \vartheta):=\int_{\Omega}\left(\frac{1}{2}|\boldsymbol{u}|^{2}+\frac{1}{2}|\nabla \varphi|^{2}+F(\varphi)+\vartheta\right)
$$

is the total energy of the system, given by the sum of the kinetic, interfacial, configuration, and thermal energies (the four summands in $\mathcal{E}$ ).

Relation (3.2), in turn, yields the following a priori estimates:

$$
\begin{aligned}
& \|\boldsymbol{u}\|_{L^{\infty}(0, T ; H)} \leq c, \\
& \|\varphi\|_{L^{\infty}(0, T ; V)} \leq c, \\
& \|\vartheta\|_{L^{\infty}\left(0, T ; L^{1}(\Omega)\right)} \leq c,
\end{aligned}
$$

where the control of the full $V$-norm of $\varphi$ (and not only of the $L^{2}$-norm of the gradient) is reached thanks to the superlinear growth of $F$ at infinity (cf. (2.8)). Note that, for getting (3.5) from (3.2), the nonnegativity of $\vartheta$ is exploited (which holds as a consequence of the approximation scheme, cf. Lemma 4.3 below). Thanks to (3.4) and Sobolev's embeddings, we also have

$$
\|\varphi\|_{L^{\infty}\left(0, T ; L^{p}(\Omega)\right)} \leq c_{p} \quad \text { for all } p \in[1, \infty) .
$$


Conservation properties. Integrating (1.2) and (1.3) over $\Omega$, and using (1.1) together with the periodic boundary conditions, we obtain

$$
\frac{\mathrm{d}}{\mathrm{d} t} \int_{\Omega} \boldsymbol{u}=\frac{\mathrm{d}}{\mathrm{d} t} \int_{\Omega} \varphi=0 \quad \text { a.e. in }(0, T) .
$$

Entropy estimate. The following estimate corresponds to the entropy production principle. It is simply obtained by testing (1.5) by $-\vartheta^{-1}$ and integrating over $\Omega$, which yields

$$
\frac{\mathrm{d}}{\mathrm{d} t} \int_{\Omega}(-\log \vartheta-\varphi)+\int_{\Omega} \frac{1}{\vartheta}\left(|\nabla \boldsymbol{u}|^{2}+|\nabla \mu|^{2}\right)+\int_{\Omega}\left(|\nabla \log \vartheta|^{2}+k_{q}\left|\nabla \vartheta^{q / 2}\right|^{2}\right)=0,
$$

where $k_{q}>0$ only depends on the exponent $q$ (cf. (2.11)). Integrating in time and recalling (3.4)-(3.5), we get the a priori bounds

$$
\begin{aligned}
& \|\log \vartheta\|_{L^{\infty}\left(0, T ; L^{1}(\Omega)\right)}+\|\log \vartheta\|_{L^{2}(0, T ; V)} \leq c, \\
& \left\|\nabla \vartheta^{q / 2}\right\|_{L^{2}(0, T ; H)} \leq c .
\end{aligned}
$$

In particular, (3.9) entails that the strict positivity (almost everywhere in $(0, T) \times \Omega)$ of $\vartheta$ is preserved also in the limit. Note also that, from (3.10), (3.5) and inequality (2.5), there follows

$$
\left\|\vartheta^{q / 2}\right\|_{L^{2}(0, T ; V)} \leq c .
$$

Now, (3.11) entails in particular

$$
\|\vartheta\|_{L^{2}(0, T ; H)} \leq c .
$$

On the other hand, arguing in a similar way as in [11, Sec. 4.2], we obtain from (3.9)-(3.10) and (3.12) the additional bound

$$
\|\vartheta\|_{L^{2}(0, T ; V)} \leq c .
$$

Temperature estimate. Using the periodic boundary conditions, we actually infer

$$
\int_{\Omega}\left(|\nabla \boldsymbol{u}|^{2}+|\nabla \mu|^{2}\right)=\frac{\mathrm{d}}{\mathrm{d} t} \int_{\Omega} \vartheta+\int_{\Omega} \vartheta\left(\varphi_{t}+\boldsymbol{u} \cdot \nabla \varphi\right)
$$

and we aim at controlling the terms on the right hand side. Actually, the first one, after integration in time, is estimated simply recalling (3.5). Using (1.3) and Hölder's and Young's inequalities, we control the second integral as follows:

$$
\int_{\Omega} \vartheta\left(\varphi_{t}+\boldsymbol{u} \cdot \nabla \varphi\right)=\int_{\Omega} \vartheta \Delta \mu=-\int_{\Omega} \nabla \vartheta \cdot \nabla \mu \leq \frac{1}{2}\left(\|\nabla \mu\|^{2}+\|\nabla \vartheta\|^{2}\right) .
$$

The first term on the right hand side is absorbed by the corresponding one on the left hand side of (3.14), while the latter is estimated thanks to (3.13). Hence, we get

$$
\begin{aligned}
& \|\boldsymbol{u}\|_{L^{2}(0, T ; V)} \leq c, \\
& \|\nabla \mu\|_{L^{2}(0, T ; H)} \leq c .
\end{aligned}
$$

Now, integrating (1.4) in space, using (3.5), (3.6) and (2.10), and taking the (essential) supremum with respect to time, we readily infer

$$
\left\|\mu_{\Omega}\right\|_{L^{\infty}(0, T)} \leq c .
$$

This property, combined with (3.17), yields

$$
\|\mu\|_{L^{2}(0, T ; V)} \leq c .
$$

It is worth noting that, even in $2 \mathrm{D}$, the above estimates, which basically correspond to those obtained in [11], do not seem sufficient to prove existence, because the right hand side of (1.5) is controlled only in $L^{1}$ (thanks to (3.16)-(3.17)). Hence, it is natural to investigate whether we can obtain some better bound exploiting the lower space dimension. For clarity, the procedure will be split into several steps. 
Remark 3.1. A further difference in our assumptions, compared to [11], stands in the present choice of a linear latent heat (in [11] we assumed to have a power-like function multiplying $\vartheta_{t}$, instead). Actually, even in 2D, it is not clear whether one could prove existence of weak solutions (i.e., solutions complying with the formulation given in [11]) in the case of a linear latent heat. Nevertheless, this assumption is allowed as one looks for strong solutions, as we are doing now. The current choice of constant specific heat, even if it yields less summability for $\vartheta$, is motivated by the fact that we need a cancellation of the terms $\vartheta_{t} \varphi_{t}$ in the forthcoming regularity estimate (cf. (3.32) and (3.34) below).

Second estimate for $\varphi$. We test (1.4) by $\Delta^{2} \varphi$ and integrate over $\Omega$. Recalling (2.10), we get

$$
\begin{aligned}
\|\nabla \Delta \varphi\|^{2} & =\int_{\Omega} F^{\prime \prime}(\varphi) \nabla \varphi \cdot \nabla \Delta \varphi-\int_{\Omega} \nabla(\vartheta+\mu) \cdot \nabla \Delta \varphi \\
& \leq c\|\nabla \Delta \varphi\|\left(\|\nabla \varphi\|+\|\varphi\|_{L^{2 p_{F}(\Omega)}}^{p_{F}}\|\nabla \varphi\|_{L^{\infty}(\Omega)}+\|\nabla \vartheta\|+\|\nabla \mu\|\right) \\
& \leq c\|\nabla \Delta \varphi\|\left(1+\|\varphi\|_{V}^{1 / 2}\|\varphi\|_{H^{3}(\Omega)}^{1 / 2}+\|\nabla \vartheta\|+\|\nabla \mu\|\right) \\
& \leq c\|\nabla \Delta \varphi\|\left(1+\|\varphi\|_{V}+\|\varphi\|_{V}^{1 / 2}\|\nabla \Delta \varphi\|^{1 / 2}+\|\nabla \vartheta\|+\|\nabla \mu\|\right) \\
& \leq \frac{1}{2}\|\nabla \Delta \varphi\|^{2}+c\left(1+\|\nabla \vartheta\|^{2}+\|\nabla \mu\|^{2}\right) .
\end{aligned}
$$

Note that (2.3) (applied to $v=\nabla \varphi$ ) and estimates (3.4), (3.6) have been used. Integrating (3.20) in time and using (3.13) and (3.17), we then obtain

$$
\|\varphi\|_{L^{2}\left(0, T ; H^{3}(\Omega)\right)} \leq c .
$$

This property has some notable consequences. Firstly, testing (1.3) by nonzero $v \in V$, recalling also (3.3), we can notice that

$$
\begin{aligned}
\left\langle\varphi_{t}, v\right\rangle & =-\int_{\Omega} \nabla \mu \cdot \nabla v-(\boldsymbol{u} \cdot \nabla \varphi, v) \leq\|\nabla \mu\|\|\nabla v\|+\|\boldsymbol{u}\|\|\nabla \varphi\|_{L^{\infty}(\Omega)}\|v\| \\
& \leq c\left(\|\nabla \mu\|+\|\varphi\|_{H^{3}(\Omega)}\right)\|v\|_{V} .
\end{aligned}
$$

Hence, dividing by $\|v\|_{V}$, passing to the supremum with respect to $v \in V \backslash\{0\}$, squaring, integrating in time, and using (3.19) and (3.21), we obtain

$$
\left\|\varphi_{t}\right\|_{L^{2}\left(0, T ; V^{\prime}\right)} \leq c .
$$

Moreover, (3.21) permits us to get a useful bound for the last term in (1.2). Indeed, using twice (2.2), we have

$$
\begin{aligned}
\|\operatorname{div}(\nabla \varphi \otimes \nabla \varphi)\| & \leq c\left\|D^{2} \varphi\right\|_{L^{4}(\Omega)}\|\nabla \varphi\|_{L^{4}(\Omega)} \leq c\|\varphi\|_{H^{3}(\Omega)}^{1 / 2}\|\varphi\|_{H^{2}(\Omega)}\|\varphi\|_{V}^{1 / 2} \\
& \leq c\|\varphi\|_{H^{3}(\Omega)}\|\varphi\|_{V} \leq c\|\varphi\|_{H^{3}(\Omega)},
\end{aligned}
$$

where the last inequality follows from (3.4). Then, squaring and integrating over $(0, T)$, thanks to $(3.21)$ we infer

$$
\|\operatorname{div}(\nabla \varphi \otimes \nabla \varphi)\|_{L^{2}(0, T ; H)} \leq c .
$$

Second estimate for $\boldsymbol{u}$. Property (3.25) allows us to apply standard regularity results to the 2D Navier-Stokes system (1.1)-(1.2) (see for instance [31, Sec. 9.6]), basically corresponding to testing (1.2) by $-\Delta \boldsymbol{u}$. As a consequence, we infer

$$
\|\boldsymbol{u}\|_{H^{1}(0, T ; H)}+\|\boldsymbol{u}\|_{L^{\infty}(0, T ; V)}+\|\boldsymbol{u}\|_{L^{2}\left(0, T ; H^{2}(\Omega)\right)} \leq c .
$$

Key estimate: $\varphi$. We start now with the key regularity estimate, which is obtained by combining in a suitable way equations (1.3), (1.4) and (1.5). At first, we deal with the Cahn-Hilliard system. Namely, we take (1.3), differentiate it with respect to time, and test the result by $\mathcal{N} \varphi_{t}$. Correspondingly, we differentiate (1.4) in time and test by $-\varphi_{t}$. Summing the obtained relations, noting that a couple of terms cancel in view of

$$
\left(\Delta \mu_{t}, \mathcal{N} \varphi_{t}\right)=-\left((-\Delta)\left(\mu_{t}-\left(\mu_{t}\right)_{\Omega}\right),(-\Delta)^{-1} \varphi_{t}\right)=-\left(\mu_{t}-\left(\mu_{t}\right)_{\Omega}, \varphi_{t}\right)=-\left(\mu_{t}, \varphi_{t}\right),
$$


where we have used also (3.7), we then get

$$
\begin{aligned}
\frac{1}{2} \frac{\mathrm{d}}{\mathrm{d} t}\left\|\varphi_{t}\right\|_{V^{\prime}}^{2}+\left\|\nabla \varphi_{t}\right\|^{2}+\int_{\Omega}\left(F^{\prime \prime}(\varphi)+\lambda\right)\left|\varphi_{t}\right|^{2} \\
\quad=\lambda\left\|\varphi_{t}\right\|^{2}-\left\langle\boldsymbol{u}_{t} \cdot \nabla \varphi, \mathcal{N} \varphi_{t}\right\rangle-\left\langle\boldsymbol{u} \cdot \nabla \varphi_{t}, \mathcal{N} \varphi_{t}\right\rangle+\left(\vartheta_{t}, \varphi_{t}\right) .
\end{aligned}
$$

Thanks to (2.9), the last term on the left hand side is nonnegative. On the other hand, we need to control the right hand side. To this aim, we first notice that, by (2.6) and (3.7), we can estimate the first term as

$$
\lambda\left\|\varphi_{t}\right\|^{2} \leq \frac{1}{8}\left\|\nabla \varphi_{t}\right\|^{2}+c\left\|\varphi_{t}\right\|_{V^{\prime}}^{2}
$$

Next, using (2.3) and standard embeddings, we infer

$$
\begin{aligned}
-\left\langle\boldsymbol{u}_{t} \cdot \nabla \varphi, \mathcal{N} \varphi_{t}\right\rangle & =\left(\left(\boldsymbol{u}_{t} \cdot \nabla \varphi, \varphi_{t}\right)\right)_{V_{0}^{\prime}} \leq\left\|\boldsymbol{u}_{t} \cdot \nabla \varphi\right\|_{V^{\prime}}\left\|\varphi_{t}\right\|_{V^{\prime}} \leq c\left\|\boldsymbol{u}_{t} \cdot \nabla \varphi\right\|\left\|\varphi_{t}\right\|_{V^{\prime}} \\
& \leq c\left\|\boldsymbol{u}_{t}\right\|\|\nabla \varphi\|_{L^{\infty}(\Omega)}\left\|\varphi_{t}\right\|_{V^{\prime}} \leq c\left\|\boldsymbol{u}_{t}\right\|^{2}+c\|\varphi\|_{H^{3}(\Omega)}^{2}\left\|\varphi_{t}\right\|_{V^{\prime}}^{2}
\end{aligned}
$$

and

$$
\begin{aligned}
-\left\langle\boldsymbol{u} \cdot \nabla \varphi_{t}, \mathcal{N} \varphi_{t}\right\rangle & =\left(\left(\boldsymbol{u} \cdot \nabla \varphi_{t}, \varphi_{t}\right)\right)_{V_{0}^{\prime}} \leq\left\|\boldsymbol{u} \cdot \nabla \varphi_{t}\right\|_{V^{\prime}}\left\|\varphi_{t}\right\|_{V^{\prime}} \leq c\left\|\boldsymbol{u} \cdot \nabla \varphi_{t}\right\|\left\|\varphi_{t}\right\|_{V^{\prime}} \\
& \leq c\|\boldsymbol{u}\|_{L^{\infty}(\Omega)}\left\|\nabla \varphi_{t}\right\|\left\|\varphi_{t}\right\|_{V^{\prime}} \leq \frac{1}{8}\left\|\nabla \varphi_{t}\right\|^{2}+c\|\boldsymbol{u}\|_{H^{2}(\Omega)}^{2}\left\|\varphi_{t}\right\|_{V^{\prime}}^{2} .
\end{aligned}
$$

In the above formulas we noted by $((\cdot, \cdot))_{V_{0}^{\prime}}$ the scalar product of $V_{0}^{\prime}$ and used the fact that $(-\Delta)$ corresponds to the Riesz operator from $V_{0}$ to $V_{0}^{\prime}$.

Hence, on account of (3.29)-(3.31), relation (3.28) takes the form

$$
\frac{1}{2} \frac{\mathrm{d}}{\mathrm{d} t}\left\|\varphi_{t}\right\|_{V^{\prime}}^{2}+\frac{3}{4}\left\|\nabla \varphi_{t}\right\|^{2}+\int_{\Omega}\left(F^{\prime \prime}(\varphi)+\lambda\right)\left|\varphi_{t}\right|^{2} \leq M_{1}(t)\left\|\varphi_{t}\right\|_{V^{\prime}}^{2}+M_{2}(t)+\left(\vartheta_{t}, \varphi_{t}\right),
$$

where the functions

$$
M_{1}(t)=c\left(1+\|\varphi\|_{H^{3}(\Omega)}^{2}+\|\boldsymbol{u}\|_{H^{2}(\Omega)}^{2}\right), \quad M_{2}(t)=c\left(1+\left\|\boldsymbol{u}_{t}\right\|^{2}\right)
$$

lie (or, more precisely, are uniformly bounded w.r.t. all approximation parameters) in $L^{1}(0, T)$ thanks to (3.21) and (3.26). It remains to control the last term on the right hand side. This, however, requires to work with the energy equation (1.5), which is our next task.

Key estimate: $\vartheta$. We start testing (1.5) by $\varphi_{t}$ in order to compute the last term in (3.32). We get

$$
\begin{gathered}
\left(\vartheta_{t}, \varphi_{t}\right)+\int_{\Omega} \vartheta \varphi_{t}^{2}=\int_{\Omega} \vartheta \boldsymbol{u} \cdot \nabla \varphi_{t}-\int_{\Omega} \vartheta \varphi_{t} \boldsymbol{u} \cdot \nabla \varphi \\
-\int_{\Omega} \kappa(\vartheta) \nabla \vartheta \cdot \nabla \varphi_{t}+\int_{\Omega}\left(|\nabla \boldsymbol{u}|^{2}+|\nabla \mu|^{2}\right) \varphi_{t},
\end{gathered}
$$

where we have used (1.1). Let us provide an estimate for the terms on the right hand side. Firstly, we have

$$
\int_{\Omega} \vartheta \boldsymbol{u} \cdot \nabla \varphi_{t} \leq\|\vartheta\|_{L^{4}(\Omega)}\|\boldsymbol{u}\|_{L^{4}(\Omega)}\left\|\nabla \varphi_{t}\right\| \leq \frac{1}{16}\left\|\nabla \varphi_{t}\right\|^{2}+c\|\vartheta\|_{V}^{2},
$$

where we have also used estimate (3.26).

Next, by (2.7) and Hölder's and Young's inequalities, thanks also to (3.4) and (3.26), we have

$$
\begin{aligned}
-\int_{\Omega} \vartheta \varphi_{t} \boldsymbol{u} \cdot \nabla \varphi & \leq\|\vartheta\|_{L^{\infty-}(\Omega)}\left\|\varphi_{t}\right\|_{L^{2+}(\Omega)}\|\boldsymbol{u}\|_{L^{\infty-}(\Omega)}\|\nabla \varphi\| \leq c\|\vartheta\| V\left\|\varphi_{t}\right\|_{L^{2+}(\Omega)} \\
& \leq c\|\vartheta\|_{V}^{2}+c\left\|\varphi_{t}\right\|_{L^{2+}(\Omega)}^{2} \leq c\|\vartheta\|_{V}^{2}+\frac{1}{16}\left\|\nabla \varphi_{t}\right\|^{2}+c\left\|\varphi_{t}\right\|_{V^{\prime}}^{2}
\end{aligned}
$$


Here (and below) the notation $\infty$ - stands for an exponent which is chosen as large (i.e. as close as infinity) as we need (in view of the fact that $V \subset L^{p}(\Omega)$ for all $p \in[1, \infty)$ ). Correspondingly, $2+$ turns out to be larger than, but close to 2. Next,

$$
-\int_{\Omega} \kappa(\vartheta) \nabla \vartheta \cdot \nabla \varphi_{t} \leq 4 \int_{\Omega} \kappa^{2}(\vartheta)|\nabla \vartheta|^{2}+\frac{1}{16}\left\|\nabla \varphi_{t}\right\|^{2} .
$$

Finally, using interpolation (cf. (2.2) and (2.6)), Young's inequality, and (3.26),

$$
\begin{aligned}
\int_{\Omega}|\nabla \boldsymbol{u}|^{2} \varphi_{t} & \leq c\left\|\varphi_{t}\right\|_{L^{4}(\Omega)}\|\nabla \boldsymbol{u}\|\|\nabla \boldsymbol{u}\|_{L^{4}(\Omega)} \leq c\left\|\varphi_{t}\right\|^{1 / 2}\left\|\nabla \varphi_{t}\right\|^{1 / 2}\|\boldsymbol{u}\|_{H^{2}(\Omega)} \\
& \leq c\left\|\varphi_{t}\right\|_{V^{\prime}}^{1 / 4}\left\|\nabla \varphi_{t}\right\|^{3 / 4}\|\boldsymbol{u}\|_{H^{2}(\Omega)} \leq c+c\|\boldsymbol{u}\|_{H^{2}(\Omega)}^{2}+c\left\|\varphi_{t}\right\|_{V^{\prime}}^{2}+\frac{1}{16}\left\|\nabla \varphi_{t}\right\|^{2} .
\end{aligned}
$$

Thanks to (3.35)-(3.38), (3.34) gives

$$
\begin{gathered}
\left(\vartheta_{t}, \varphi_{t}\right)+\int_{\Omega} \vartheta \varphi_{t}^{2} \leq c\left(1+\|\boldsymbol{u}\|_{H^{2}(\Omega)}^{2}+\|\vartheta\|_{V}^{2}+\left\|\varphi_{t}\right\|_{V^{\prime}}^{2}\right) \\
+\frac{1}{4}\left\|\nabla \varphi_{t}\right\|^{2}+4 \int_{\Omega} \kappa^{2}(\vartheta)|\nabla \vartheta|^{2}+\int_{\Omega}|\nabla \mu|^{2} \varphi_{t} .
\end{gathered}
$$

The last two terms on the right hand side are still to be controlled. The quadratic term in $\nabla \mu$, which is the most difficult one, will be dealt with at the end. In order to treat the term in $\nabla \vartheta$, we need another estimate. Namely, we test (1.5) by $8 K(\vartheta)$ (cf. (2.12)). Let us set

$$
\mathcal{J}(r):=\int_{0}^{r} K(s) \mathrm{d} s=\frac{r^{2}}{2}+\frac{1}{(q+1)(q+2)} r^{q+2}, \quad r \geq 0 .
$$

Then, we obtain

$$
\begin{aligned}
& 8 \frac{\mathrm{d}}{\mathrm{d} t} \int_{\Omega} \mathcal{J}(\vartheta)+8 \int_{\Omega} \boldsymbol{u} \cdot \nabla \mathcal{J}(\vartheta)+8 \int_{\Omega} \kappa^{2}(\vartheta)|\nabla \vartheta|^{2} \\
& \quad=-8 \int_{\Omega} \vartheta K(\vartheta)\left(\varphi_{t}+\boldsymbol{u} \cdot \nabla \varphi\right)+8 \int_{\Omega} K(\vartheta)\left(|\nabla \boldsymbol{u}|^{2}+|\nabla \mu|^{2}\right),
\end{aligned}
$$

where, in fact, the second integral on the left hand side is zero in view of (1.1) and the periodic boundary conditions. Again, we need to provide an estimate for the terms on the right hand side. At first, recalling (2.12) and using (2.6), we have

$$
\begin{aligned}
-8 \int_{\Omega} \vartheta K(\vartheta) \varphi_{t} & \leq c \int_{\Omega}\left(\vartheta^{2}+\vartheta^{q+2}\right)\left|\varphi_{t}\right| \leq c \int_{\Omega}\left(1+\vartheta^{q+2}\right)\left|\varphi_{t}\right| \\
& \leq c\left\|\varphi_{t}\right\|+\int_{\Omega} \vartheta^{q+2}\left|\varphi_{t}\right| \leq c+\frac{1}{16}\left\|\nabla \varphi_{t}\right\|^{2}+c\left\|\varphi_{t}\right\|_{V^{\prime}}^{2}+c \int_{\Omega} \vartheta^{q+2}\left|\varphi_{t}\right| .
\end{aligned}
$$

The last term needs to be managed accurately. We start by noting that

$$
c \int_{\Omega} \vartheta^{q+2}\left|\varphi_{t}\right| \leq c\left\|\vartheta^{q+2}\right\|\left\|\varphi_{t}\right\| \leq c\|\vartheta\|_{L^{2 q+4}(\Omega)}^{q+2}\left\|\varphi_{t}\right\|_{V^{\prime}}^{1 / 2}\left\|\nabla \varphi_{t}\right\|^{1 / 2} .
$$

Next, we observe that, using (2.4) with the choices $r=2 q+4$ and $s=1$, we would obtain

$$
\|\vartheta\|_{L^{2 q+4}(\Omega)} \leq\|\vartheta\|_{L^{1}(\Omega)}^{\frac{1}{2 q+4}}\|\vartheta\|_{L^{\infty}(\Omega)}^{\frac{2 q+3}{2 q+4}}
$$


However the above interpolation exponents do not work in our case and need to be modified slightly. Actually, recalling also (3.5), we can continue (3.43) as follows:

$$
\begin{aligned}
c \int_{\Omega} \vartheta^{q+2}\left|\varphi_{t}\right| & \leq c\left(\|\vartheta\|_{L^{1}(\Omega)}^{\frac{1}{2 q+4}-}\|\vartheta\|_{L^{\infty-}(\Omega)}^{\frac{2 q+3}{2 q+4}+}\right)^{q+2}\left\|\varphi_{t}\right\|_{V^{\prime}}^{1 / 2}\left\|\nabla \varphi_{t}\right\|^{1 / 2} \\
& \leq c\|\vartheta\|_{L^{\infty-}(\Omega)}^{\frac{2 q+3}{2}+}\left\|\varphi_{t}\right\|_{V^{\prime}}^{1 / 2}\left\|\nabla \varphi_{t}\right\|^{1 / 2} \leq c\left\|\vartheta^{q+1}\right\|_{L^{\infty-}(\Omega)}^{\frac{2 q+3}{2 q+2}+}\left\|\varphi_{t}\right\|_{V^{\prime}}^{1 / 2}\left\|\nabla \varphi_{t}\right\|^{1 / 2} .
\end{aligned}
$$

As above, $\infty-$ stands for an exponent $P \in\left[1, \infty\right.$ ) (which we can choose as large as we need) and the number $\frac{2 q+3}{2}+$ depends on the choice of $P$ and will be closer to $\frac{2 q+3}{2}$ as larger is taken $P$. The same applies to $\frac{2 q+3}{2 q+2}+$ and other exponents below. Of course, also the constants $c$ will depend on the choice of $P$ (and will be larger for larger $P$ ). Then, recalling (2.5) and subsequently using Young's inequality with exponents 8 , 4, and 8/5, computation (3.45) can be continued this way:

$$
\begin{aligned}
c \int_{\Omega} \vartheta^{q+2}\left|\varphi_{t}\right| & \leq c\left\|\varphi_{t}\right\|_{V^{\prime}}^{1 / 2}\left\|\nabla \varphi_{t}\right\|^{1 / 2}\left(1+\left\|\nabla \vartheta^{q+1}\right\|^{\frac{2 q+3}{2 q+2}+}\right) \\
& \leq c+c\left\|\varphi_{t}\right\|_{V^{\prime}}^{4}+\frac{1}{16}\left\|\nabla \varphi_{t}\right\|^{2}+c\left\|\nabla \vartheta^{q+1}\right\|^{\frac{4(2 q+3)}{5(q+1)}+},
\end{aligned}
$$

and, thanks to (2.11) (actually, $q>1$ would be enough at this level), we can take $P$ so large that $\frac{4(2 q+3)}{5(q+1)}+$ is strictly smaller than 2 . Hence, using Young's inequality again, and recalling (2.13), we conclude that

$$
\begin{aligned}
-8 \int_{\Omega} \vartheta K(\vartheta) \varphi_{t} & \leq c+\frac{1}{8}\left\|\nabla \varphi_{t}\right\|^{2}+c\left\|\varphi_{t}\right\|_{V^{\prime}}^{2}+c\left\|\varphi_{t}\right\|_{V^{\prime}}^{4}+c\left\|\nabla \vartheta^{q+1}\right\|^{\frac{4(2 q+3)}{5(q+1)}+} \\
& \leq c+\frac{1}{8}\left\|\nabla \varphi_{t}\right\|^{2}+c\left\|\varphi_{t}\right\|_{V^{\prime}}^{4}+\int_{\Omega} \kappa^{2}(\vartheta)|\nabla \vartheta|^{2} .
\end{aligned}
$$

The estimation of the subsequent summand in (3.41) is simpler. Actually, recalling also (3.4), (3.5) and (3.26), and using once more (2.5), (2.13) and Young's inequality, we get

$$
\begin{aligned}
-8 \int_{\Omega} \vartheta K(\vartheta) \boldsymbol{u} \cdot \nabla \varphi & \leq c \int_{\Omega}\left(1+\vartheta^{q+2}\right)|\boldsymbol{u}||\nabla \varphi| \leq c\left(1+\left\|\vartheta^{q+2}\right\|_{L^{2+}(\Omega)}\right)\|\boldsymbol{u}\|_{L^{\infty-}(\Omega)}\|\nabla \varphi\| \\
& \leq c\left(1+\left\|\vartheta^{q+1}\right\|_{L^{\frac{q+2}{q+1}}}^{\frac{2(q+2)}{q+1}+}{ }_{(\Omega)}\right) \leq c\left(1+\left\|\nabla \vartheta^{q+1}\right\|^{\frac{q+2}{q+1}}\right) \leq c+\int_{\Omega} \kappa^{2}(\vartheta)|\nabla \vartheta|^{2},
\end{aligned}
$$

since, clearly, $\frac{q+2}{q+1}<2$. Next, recalling (3.26) and (2.16) and arguing analogously to (3.38), we have

$$
8 \int_{\Omega}|\nabla \boldsymbol{u}|^{2} K(\vartheta) \leq c\|K(\vartheta)\|_{L^{4}(\Omega)}\|\nabla \boldsymbol{u}\|\|\nabla \boldsymbol{u}\|_{L^{4}(\Omega)} \leq c+c\|\boldsymbol{u}\|_{H^{2}(\Omega)}^{2}+\int_{\Omega} \kappa^{2}(\vartheta)|\nabla \vartheta|^{2} .
$$

Collecting (3.42)-(3.49), (3.41) gives

$$
\begin{aligned}
& 8 \frac{\mathrm{d}}{\mathrm{d} t} \int_{\Omega} \mathcal{J}(\vartheta)+5 \int_{\Omega} \kappa^{2}(\vartheta)|\nabla \vartheta|^{2} \\
& \quad \leq c+c\|\boldsymbol{u}\|_{H^{2}(\Omega)}^{2}+\frac{1}{8}\left\|\nabla \varphi_{t}\right\|^{2}+c\left\|\varphi_{t}\right\|_{V^{\prime}}^{4}+8 \int_{\Omega} K(\vartheta)|\nabla \mu|^{2} .
\end{aligned}
$$

Summing (3.39) and (3.50) we then get 


$$
\begin{aligned}
& \left(\vartheta_{t}, \varphi_{t}\right)+\int_{\Omega} \vartheta \varphi_{t}^{2}+8 \frac{\mathrm{d}}{\mathrm{d} t} \int_{\Omega} \mathcal{J}(\vartheta)+\int_{\Omega} \kappa^{2}(\vartheta)|\nabla \vartheta|^{2} \\
& \leq c\left(1+\|\boldsymbol{u}\|_{H^{2}(\Omega)}^{2}+\|\vartheta\|_{V}^{2}+\left\|\varphi_{t}\right\|_{V^{\prime}}^{4}\right)+\frac{3}{8}\left\|\nabla \varphi_{t}\right\|^{2}+\int_{\Omega}\left(8 K(\vartheta)+\varphi_{t}\right)|\nabla \mu|^{2} .
\end{aligned}
$$

Hence, summing (3.32) and (3.51) we obtain

$$
\begin{aligned}
& \frac{1}{2} \frac{\mathrm{d}}{\mathrm{d} t}\left\|\varphi_{t}\right\|_{V^{\prime}}^{2}+8 \frac{\mathrm{d}}{\mathrm{d} t} \int_{\Omega} \mathcal{J}(\vartheta)+\frac{3}{8}\left\|\nabla \varphi_{t}\right\|^{2}+\int_{\Omega}\left(F^{\prime \prime}(\varphi)+\lambda\right)\left|\varphi_{t}\right|^{2} \\
& \quad+\int_{\Omega} \vartheta \varphi_{t}^{2}+\int_{\Omega} \kappa^{2}(\vartheta)|\nabla \vartheta|^{2} \leq M_{1}(t)\left\|\varphi_{t}\right\|_{V^{\prime}}^{2}+M_{2}(t) \\
& \quad+c\left(1+\|\boldsymbol{u}\|_{H^{2}(\Omega)}^{2}+\|\vartheta\|_{V}^{2}+\left\|\varphi_{t}\right\|_{V^{\prime}}^{4}\right)+\int_{\Omega}\left(8 K(\vartheta)+\varphi_{t}\right)|\nabla \mu|^{2} .
\end{aligned}
$$

Neglecting some positive terms on the left hand side and rearranging, we then arrive at

$$
\begin{gathered}
\frac{1}{2} \frac{\mathrm{d}}{\mathrm{d} t}\left\|\varphi_{t}\right\|_{V^{\prime}}^{2}+8 \frac{\mathrm{d}}{\mathrm{d} t} \int_{\Omega} \mathcal{J}(\vartheta)+\frac{3}{8}\left\|\nabla \varphi_{t}\right\|^{2}+\int_{\Omega} \kappa^{2}(\vartheta)|\nabla \vartheta|^{2} \\
\leq M_{3}(t)\left\|\varphi_{t}\right\|_{V^{\prime}}^{2}+M_{4}(t)+\int_{\Omega}\left(8 K(\vartheta)+\varphi_{t}\right)|\nabla \mu|^{2},
\end{gathered}
$$

where we have set

$$
M_{3}(t)=c\left(1+\|\varphi\|_{H^{3}(\Omega)}^{2}+\|\boldsymbol{u}\|_{H^{2}(\Omega)}^{2}+\left\|\varphi_{t}\right\|_{V^{\prime}}^{2}\right), \quad M_{4}(t)=c\left(1+\left\|\boldsymbol{u}_{t}\right\|^{2}+\|\boldsymbol{u}\|_{H^{2}(\Omega)}^{2}+\|\vartheta\|_{V}^{2}\right) .
$$

Key estimate: quadratic terms. The most difficult part of our argument concerns the control of the last term on the right hand side of (3.53). This is based on the embedding inequality (A.1) proved in Lemma A.1 below. Indeed, applying (A.1) to $\xi=|\nabla \mu|^{2}$ and using once more (2.16) together with (3.7) and the Poincaré-Wirtinger inequality, we get

$$
\begin{aligned}
\int_{\Omega} & \left(8 K(\vartheta)+\varphi_{t}\right)|\nabla \mu|^{2} \leq c\left(\|K(\vartheta)\|_{V}+\left\|\nabla \varphi_{t}\right\|\right)\left\||\nabla \mu|^{2}\right\|_{V^{\prime}} \\
& \leq c+\frac{1}{2} \int_{\Omega} \kappa^{2}(\vartheta)|\nabla \vartheta|^{2}+\frac{1}{8}\left\|\nabla \varphi_{t}\right\|^{2}+c\left\||\nabla \mu|^{2}\right\|_{V^{\prime}}^{2} \\
& \leq c+\frac{1}{2} \int_{\Omega} \kappa^{2}(\vartheta)|\nabla \vartheta|^{2}+\frac{1}{8}\left\|\nabla \varphi_{t}\right\|^{2}+c\left\||\nabla \mu|^{2}\right\|_{L^{1}(\Omega)}^{2} \log \left(e+\left\||\nabla \mu|^{2}\right\|_{L^{2}(\Omega)}\right) .
\end{aligned}
$$

Now, let us notice that $\psi(r)=e^{r}, r \in \mathbb{R}$ and $\psi^{*}(s)=s(\log s-1), s>0$ (extended by continuity to $s=0$ by setting $\psi^{*}(0)=0$ ) are convex conjugate functions. Consequently, for any $r \in \mathbb{R}, s \geq 0$, we have (cf., e.g., [6, Sec. 1.4]) $r s \leq \psi(r)+\psi^{*}(s)$. Applying this property to $r=\log \left(e+\left\||\nabla \mu|^{2}\right\|_{L^{2}(\Omega)}\right)$ and $s=c\left\||\nabla \mu|^{2}\right\|_{L^{1}(\Omega)}^{2}$, the last term (let us note it as $\mathcal{I})$ in $(3.55)$ can be controlled as follows:

$$
\begin{aligned}
\mathcal{I} & \leq c\left\||\nabla \mu|^{2}\right\|_{L^{1}(\Omega)}^{2}\left(\log \left(c\left\||\nabla \mu|^{2}\right\|_{L^{1}(\Omega)}^{2}\right)-1\right)+e+\left\||\nabla \mu|^{2}\right\|_{L^{2}(\Omega)} \\
& \leq c+c\|\nabla \mu\|^{4} \log \left(e+\|\nabla \mu\|^{2}\right)+\|\nabla \mu\|_{L^{4}(\Omega)}^{2},
\end{aligned}
$$

where, observing that $\left\||\nabla \mu|^{2}\right\|_{L^{1}(\Omega)}^{2}=\|\nabla \mu\|^{4}$, we used the fact that 


$$
\begin{aligned}
\left\||\nabla \mu|^{2}\right\|_{L^{1}(\Omega)}^{2} \log \left(c\left\||\nabla \mu|^{2}\right\|_{L^{1}(\Omega)}^{2}\right) & =\|\nabla \mu\|^{4} \log \left(c\|\nabla \mu\|^{4}\right) \\
& =\|\nabla \mu\|^{4}\left(\log c+2 \log \|\nabla \mu\|^{2}\right) \\
& \leq c\|\nabla \mu\|^{4}\left(1+\log \|\nabla \mu\|^{2}\right) \\
& \leq c\|\nabla \mu\|^{4} \log \left(e+\|\nabla \mu\|^{2}\right),
\end{aligned}
$$

where we used the elementary inequality $1+\log \lambda \leq c \log (e+\lambda)$, holding for $\lambda>0$. Now, to manage the last term of (3.56), we use equation (1.3), estimate (3.4), and inequalities (2.2) and (2.6):

$$
\begin{aligned}
\|\nabla \mu\|_{L^{4}(\Omega)}^{2} & \leq c\|\nabla \mu\|\|\mu\|_{H^{2}(\Omega)} \leq c\|\nabla \mu\|\left(\|\mu\|_{V}+\|\Delta \mu\|\right) \leq c\|\mu\|_{V}^{2}+c\left\|\varphi_{t}\right\|^{2}+c\|\boldsymbol{u} \cdot \nabla \varphi\|^{2} \\
& \leq c\|\mu\|_{V}^{2}+\frac{1}{8}\left\|\nabla \varphi_{t}\right\|^{2}+c\left\|\varphi_{t}\right\|_{V^{\prime}}^{2}+c\|\boldsymbol{u}\|_{L^{\infty}(\Omega)}^{2}\|\nabla \varphi\|^{2} \\
& \leq c\|\mu\|_{V}^{2}+\frac{1}{8}\left\|\nabla \varphi_{t}\right\|^{2}+c\left\|\varphi_{t}\right\|_{V^{\prime}}^{2}+c\|\boldsymbol{u}\|_{H^{2}(\Omega)}^{2} .
\end{aligned}
$$

On the other hand, the first nonconstant term on the right hand side of (3.56) needs a further manipulation. Namely, we have to test equation (1.3) by $-\mu$. Then, noting that both terms on the left hand side of (1.3) have zero spatial mean and using the Poincaré-Wirtinger inequality, we get

$$
\begin{aligned}
\|\nabla \mu\|^{2} & =-\int_{\Omega}\left(\varphi_{t}+\boldsymbol{u} \cdot \nabla \varphi\right) \mu=-\int_{\Omega}\left(\varphi_{t}+\boldsymbol{u} \cdot \nabla \varphi\right)\left(\mu-\mu_{\Omega}\right) \leq\left\|\mu-\mu_{\Omega}\right\| V\left\|\varphi_{t}+\boldsymbol{u} \cdot \nabla \varphi\right\|_{V^{\prime}} \\
& \leq \frac{1}{2}\|\nabla \mu\|^{2}+c\left\|\varphi_{t}\right\|_{V^{\prime}}^{2}+c\|\boldsymbol{u} \cdot \nabla \varphi\|_{V^{\prime}}^{2} \leq \frac{1}{2}\|\nabla \mu\|^{2}+c\left\|\varphi_{t}\right\|_{V^{\prime}}^{2}+c\|\boldsymbol{u} \cdot \nabla \varphi\|_{L^{4 / 3}(\Omega)}^{2} \\
& \leq \frac{1}{2}\|\nabla \mu\|^{2}+c\left\|\varphi_{t}\right\|_{V^{\prime}}^{2}+c\|\boldsymbol{u}\|_{L^{4}(\Omega)}^{2}\|\nabla \varphi\|^{2} \leq \frac{1}{2}\|\nabla \mu\|^{2}+c\left\|\varphi_{t}\right\|_{V^{\prime}}^{2}+c,
\end{aligned}
$$

where in the last line we also used (3.26). Consequently,

$$
\begin{aligned}
c\|\nabla \mu\|^{4} \log \left(e+\|\nabla \mu\|^{2}\right) & \leq c\left(1+\left\|\varphi_{t}\right\|_{V^{\prime}}^{4}\right) \log \left(e+c\left(1+\left\|\varphi_{t}\right\|_{V^{\prime}}^{2}\right)\right) \\
& \leq c+c\left\|\varphi_{t}\right\|_{V^{\prime}}^{4} \log \left(e+\left\|\varphi_{t}\right\|_{V^{\prime}}^{2}\right) .
\end{aligned}
$$

Hence, collecting (3.56)-(3.60), (3.55) gives

$$
\begin{aligned}
& \int_{\Omega}\left(8 K(\vartheta)+\varphi_{t}\right)|\nabla \mu|^{2} \leq c+\frac{1}{2} \int_{\Omega} \kappa^{2}(\vartheta)|\nabla \vartheta|^{2}+\frac{1}{4}\left\|\nabla \varphi_{t}\right\|^{2}+c\left\|\varphi_{t}\right\|_{V^{\prime}}^{2}+c\|\mu\|_{V}^{2} \\
& \quad+c\|\boldsymbol{u}\|_{H^{2}(\Omega)}^{2}+c\left\|\varphi_{t}\right\|_{V^{\prime}}^{4} \log \left(e+\left\|\varphi_{t}\right\|_{V^{\prime}}^{2}\right) .
\end{aligned}
$$

Plugging (3.61) into (3.53) we then get

$$
\begin{gathered}
\frac{1}{2} \frac{\mathrm{d}}{\mathrm{d} t}\left\|\varphi_{t}\right\|_{V^{\prime}}^{2}+8 \frac{\mathrm{d}}{\mathrm{d} t} \int_{\Omega} \mathcal{J}(\vartheta)+\frac{1}{8}\left\|\nabla \varphi_{t}\right\|^{2}+\frac{1}{2} \int_{\Omega} \kappa^{2}(\vartheta)|\nabla \vartheta|^{2} \\
\leq M_{5}(t)\left\|\varphi_{t}\right\|_{V^{\prime}}^{2}+M_{6}(t)+c\left\|\varphi_{t}\right\|_{V^{\prime}}^{4} \log \left(e+\left\|\varphi_{t}\right\|_{V^{\prime}}^{2}\right),
\end{gathered}
$$

where

$$
\begin{aligned}
& M_{5}(t)=c\left(1+\|\varphi\|_{H^{3}(\Omega)}^{2}+\|\boldsymbol{u}\|_{H^{2}(\Omega)}^{2}+\left\|\varphi_{t}\right\|_{V^{\prime}}^{2}\right), \\
& M_{6}(t)=c\left(1+\left\|\boldsymbol{u}_{t}\right\|^{2}+\|\boldsymbol{u}\|_{H^{2}(\Omega)}^{2}+\|\vartheta\|_{V}^{2}+\|\mu\|_{V}^{2}\right) .
\end{aligned}
$$

Let us now set

$$
Y_{1}(t):=\frac{1}{2}\left\|\varphi_{t}(t)\right\|_{V^{\prime}}^{2}, \quad Y_{2}(t):=8 \int_{\Omega} \mathcal{J}(\vartheta(t)) .
$$


Then, from (3.61) we obtain the following differential inequality:

$$
Y_{1}^{\prime}(t)+Y_{2}^{\prime}(t) \leq M_{5}(t) Y_{1}(t)+M_{6}(t)+c Y_{1}^{2}(t) \log \left(e+Y_{1}(t)\right) .
$$

Setting $Z(t):=e+Y_{1}(t)+Y_{2}(t)$, and dividing both hand sides of (3.66) by $Z \log Z$, it is then easy to get

$$
\frac{\mathrm{d}}{\mathrm{d} t} \log \log Z(t)=\frac{Z^{\prime}(t)}{Z(t) \log Z(t)} \leq M_{5}(t)+\frac{M_{6}(t)}{Z(t) \log Z(t)}+c Y_{1}(t) .
$$

Now, let us notice that, in view of the a-priori estimates (3.11), (3.13), (3.19), (3.21), (3.23), and (3.26), we have

$$
\left\|Y_{1}\right\|_{L^{1}(0, T)}+\left\|M_{5}\right\|_{L^{1}(0, T)}+\left\|M_{6}\right\|_{L^{1}(0, T)} \leq c .
$$

Moreover, we can notice that, at least formally (actually at the approximate level this is justified once the approximate $\varphi_{t}$ lies in $C^{0}\left([0, T] ; V^{\prime}\right)$, cf. also Remark 4.7 below),

$$
\begin{aligned}
Z(0) & =e+\frac{1}{2}\left\|\varphi_{t}(0)\right\|_{V^{\prime}}^{2}+8 \int_{\Omega} \mathcal{J}\left(\vartheta_{0}\right) \\
& \leq c+c\|\Delta \mu(0)\|_{V^{\prime}}^{2}+c\left\|\boldsymbol{u}_{0} \cdot \nabla \varphi_{0}\right\|_{V^{\prime}}^{2}+c \int_{\Omega} \mathcal{J}\left(\vartheta_{0}\right) \\
& \leq c+\frac{1}{2}\|\mu(0)\|_{V}^{2}+c\left\|\boldsymbol{u}_{0}\right\|_{V}^{2}\left\|\varphi_{0}\right\|_{H^{3}(\Omega)}^{2}+c\left\|\vartheta_{0}\right\|^{2}+c\left\|\vartheta_{0}\right\|_{L^{q+2}(\Omega)}^{q+2} \\
& \leq c+c\left\|\Delta \varphi_{0}\right\|_{V}^{2}+c\left\|F^{\prime}\left(\varphi_{0}\right)\right\|_{V}^{2}+c\left\|\vartheta_{0}\right\|_{V}^{q+2}<\infty,
\end{aligned}
$$

where in the second row we used equation (1.3), in the third we used standard interpolation and embeddings and the definition of $\mathcal{J}$, and in the fourth we used equation (1.4) and the assumptions (2.10) on the potential and (2.17)-(2.19) on the initial data.

Hence, thanks to (3.68) and to (3.69), we can integrate (3.67) over $(0, T)$ to obtain

$$
\|Z\|_{L^{\infty}(0, T)} \leq c .
$$

In particular, as often happens in 2D models, $Z$ turns out to grow with respect to time (at most) as fast as a double exponential; however it does not explode in finite times. This is the key point in our existence proof.

Key estimate: consequences. Recalling also (3.40), (3.70) gives

$$
\begin{aligned}
& \left\|\varphi_{t}\right\|_{L^{\infty}\left(0, T ; V^{\prime}\right)} \leq c, \\
& \|\vartheta\|_{L^{\infty}\left(0, T ; L^{q+2}(\Omega)\right)} \leq c .
\end{aligned}
$$

Using (1.3) we can estimate, as in (3.59), the $H$-norm of $\nabla \mu$ in terms of the $V^{\prime}$-norm of $\varphi_{t}$. Hence, recalling also (3.18), we get

$$
\|\mu\|_{L^{\infty}(0, T ; V)} \leq c .
$$

With these properties at disposal, we can go back to relation (3.62). Integrating it over $(0, T)$, we infer (cf. also (2.12), (3.7))

$$
\begin{aligned}
& \left\|\varphi_{t}\right\|_{L^{2}(0, T ; V)} \leq c, \\
& \|K(\vartheta)\|_{L^{2}(0, T ; V)} \leq c .
\end{aligned}
$$

In addition to that, an easy interpolation argument and estimates (3.21), (3.26) permit us to check that

$$
\|\boldsymbol{u} \cdot \nabla \varphi\|_{L^{2}(0, T ; V)} \leq c\|\boldsymbol{u}\|_{L^{\infty}(0, T ; V)}\|\varphi\|_{L^{2}\left(0, T ; H^{3}(\Omega)\right)} \leq c .
$$

Hence, viewing (1.3) as a time-dependent family of elliptic problems and using standard regularity results with (3.76) and (3.74), we infer

$$
\|\mu\|_{L^{2}\left(0, T ; H^{3}(\Omega)\right)} \leq c .
$$


Estimate of $\mu_{t}$ and $\vartheta_{t}$. In order to pass to the limit in the nonlinear terms involving $\vartheta$ and $\mu$ we will apply the Aubin-Lions lemma. To this aim, we need to deduce some a-priori estimates on $\mu_{t}$ and $\vartheta_{t}$. Hence, we (formally) differentiate (1.4) with respect to time and use (1.5) to get

$$
\mu_{t}=-\Delta \varphi_{t}+F^{\prime \prime}(\varphi) \varphi_{t}+\boldsymbol{u} \cdot \nabla \vartheta+\vartheta\left(\varphi_{t}+\boldsymbol{u} \cdot \nabla \varphi\right)-\Delta K(\vartheta)-|\nabla \boldsymbol{u}|^{2}-|\nabla \mu|^{2} .
$$

For a justification of this procedure we refer the reader to Remark 4.7 below. Let us test the above relation by nonzero $v \in V$. We get, using our choice of boundary conditions,

$$
\begin{aligned}
& \left\langle\mu_{t}, v\right\rangle=\int_{\Omega} \nabla\left(\varphi_{t}+K(\vartheta)\right) \cdot \nabla v+\left(F^{\prime \prime}(\varphi) \varphi_{t}+\boldsymbol{u} \cdot \nabla \vartheta+\vartheta\left(\varphi_{t}+\boldsymbol{u} \cdot \nabla \varphi\right)-|\nabla \boldsymbol{u}|^{2}-|\nabla \mu|^{2}, v\right) \\
& \leq\|v\|_{V}\left(\left\|\nabla\left(\varphi_{t}+K(\vartheta)\right)\right\|\right. \\
& \left.\quad+\left\|F^{\prime \prime}(\varphi) \varphi_{t}+\boldsymbol{u} \cdot \nabla \vartheta+\vartheta\left(\varphi_{t}+\boldsymbol{u} \cdot \nabla \varphi\right)-|\nabla \boldsymbol{u}|^{2}-|\nabla \mu|^{2}\right\|_{L^{3 / 2}(\Omega)}\right) .
\end{aligned}
$$

Dividing by $\|v\|$, passing to the supremum with respect to $v \in V \backslash\{0\}$, squaring, and integrating in time, we would then obtain

$$
\|\mu\|_{H^{1}\left(0, T ; V^{\prime}\right)} \leq c,
$$

provided we could prove that

$$
\begin{aligned}
& \left\|\nabla\left(\varphi_{t}+K(\vartheta)\right)\right\|_{L^{2}(0, T ; H)} \leq c, \\
& \left\|F^{\prime \prime}(\varphi) \varphi_{t}+\boldsymbol{u} \cdot \nabla \vartheta+\vartheta\left(\varphi_{t}+\boldsymbol{u} \cdot \nabla \varphi\right)-|\nabla \boldsymbol{u}|^{2}-|\nabla \mu|^{2}\right\|_{L^{2}\left(0, T ; L^{3 / 2}(\Omega)\right)} \leq c,
\end{aligned}
$$

where the exponent $3 / 2$ is chosen just for simplicity (any number strictly greater than 1 would be allowed, indeed). Now, (3.81) is an immediate consequence of (3.74)-(3.75), whereas (3.82) follows by appropriately combining all the above a-priori estimates and using standard inequalities. The details are lengthy but straightforward; hence, they are left to the reader.

To conclude, we test equation (1.5) by $v \in V \backslash\{0\}$. Performing the very same computations as above we then get

$$
\|\vartheta\|_{H^{1}\left(0, T ; V^{\prime}\right)} \leq c \text {. }
$$

This is the last estimate we need.

Weak sequential stability. We assume to have a sequence of solutions $\left(\boldsymbol{u}_{n}, \varphi_{n}, \mu_{n}, \vartheta_{n}\right)$ satisfying the a-priori estimates obtained above uniformly with respect to $n$. This could be, for instance, a sequence of approximate solutions provided by the fixed-point argument performed in the next section. Then, we aim at proving that, up to the extraction of a subsequence, we can find a limit quadruple $(\boldsymbol{u}, \varphi, \mu, \vartheta)$ satisfying (1.1)-(1.5) in the sense of Theorem 2.1. Note that, in view of the uniform character of the estimates, even though the approximate solutions are defined only locally in time, we will have global solutions in the limit (cf. also Remark 4.5 below). For this reason, and also for the sake of simplicity, we shall directly work on the original reference time interval $(0, T)$.

That said, the above a-priori estimates (cf., in particular, (3.13), (3.21), (3.26), (3.71)-(3.75),(3.77), (3.80), and (3.83)), together with standard weak compactness results, entail

$$
\begin{array}{cl}
\boldsymbol{u}_{n} \rightarrow \boldsymbol{u} & \text { weakly star in } H^{1}(0, T ; H) \cap L^{\infty}(0, T ; V) \cap L^{2}\left(0, T ; H^{2}(\Omega)\right), \\
\varphi_{n} \rightarrow \varphi & \text { weakly star in } W^{1, \infty}\left(0, T ; V^{\prime}\right) \cap H^{1}(0, T ; V) \cap L^{2}\left(0, T ; H^{3}(\Omega)\right), \\
\mu_{n} \rightarrow \mu & \text { weakly star in } H^{1}\left(0, T ; V^{\prime}\right) \cap L^{\infty}(0, T ; V) \cap L^{2}\left(0, T ; H^{3}(\Omega)\right), \\
\vartheta_{n} \rightarrow \vartheta & \text { weakly star in } H^{1}\left(0, T ; V^{\prime}\right) \cap L^{\infty}\left(0, T ; L^{q+2}(\Omega)\right) \cap L^{2}(0, T ; V) .
\end{array}
$$

Here and below, all convergence relations are intended to hold up to the extraction of non-relabelled subsequences. Note also that (3.85), by interpolation (cf., e.g., [7, formula (2.5.38)]), yields

$$
\varphi_{n} \rightarrow \varphi \quad \text { weakly in } H^{s}\left(0, T ; H^{3-2 s}(\Omega)\right) \text { for all } s \in[0,1] .
$$

Then, the Aubin-Lions compactness theorem (cf. [26, p. 58] or [33, Cor. 4, Sec. 8]) entails (actually something more is true) 


$$
\begin{array}{ll}
\boldsymbol{u}_{n} \rightarrow \boldsymbol{u} & \text { strongly in } C^{0}([0, T] ; H) \cap L^{2}(0, T ; V), \\
\varphi_{n} \rightarrow \varphi & \text { strongly in } C^{0}([0, T] ; V) \cap L^{2}\left(0, T ; H^{2}(\Omega)\right), \\
\mu_{n} \rightarrow \mu & \text { strongly in } C^{0}([0, T] ; H) \cap L^{2}\left(0, T ; H^{2}(\Omega)\right), \\
\vartheta_{n} \rightarrow \vartheta & \text { strongly in } C^{0}\left([0, T] ; V^{\prime}\right) \cap L^{2}(0, T ; H) .
\end{array}
$$

Notice that, to deduce the first (3.90), also (3.88) has been used together with the compact embedding $H^{s}(0, T) \subset \subset$ $C^{0}([0, T])$ (or, more precisely, its vector-valued analogue), holding for $s>1 / 2$.

We now claim that the above relations suffice to take the limit $n \nearrow \infty$ in all equations of our system (1.1)-(1.5). To see this, we limit ourselves to consider the most troublesome nonlinear terms, the other ones being in fact almost straightforward to treat. To start with, we note that

$$
\nabla \varphi_{n} \otimes \nabla \varphi_{n} \rightarrow \nabla \varphi \otimes \nabla \varphi \quad \text { strongly in } C^{0}\left([0, T] ; L^{1}(\Omega)\right)
$$

thanks to (3.90). Actually, proceeding as in (3.24)-(3.25), we can check that $\operatorname{div}(\nabla \varphi \otimes \nabla \varphi)$ lies in $L^{2}(0, T ; H)$, which allows for (1.2) to hold pointwise (almost everywhere). Next, we notice that (3.92) entails $\vartheta_{n} \rightarrow \vartheta$ a.e. in $(0, T) \times \Omega$. Such a property, combined with (3.72), (3.75), and a generalized version of Lebesgue's theorem, gives

$$
\vartheta_{n} \rightarrow \vartheta \quad \text { strongly in } L^{p}\left(0, T ; L^{(q+2)-}(\Omega)\right) \cap L^{(2 q+2)-}\left(0, T ; L^{p}(\Omega)\right) \text { for all } p \in[1, \infty) .
$$

In particular, recalling (2.12), we get

$$
K\left(\vartheta_{n}\right) \rightarrow K(\vartheta) \quad \text { strongly in } L^{p}((0, T) \times \Omega) \text { for a suitable } p>1 .
$$

Hence,

$$
-\operatorname{div}\left(\kappa\left(\vartheta_{n}\right) \nabla \vartheta_{n}\right)=-\Delta K\left(\vartheta_{n}\right) \rightarrow-\Delta K(\vartheta)
$$

at least in the sense of distributions. More precisely, the limit function $K(\vartheta)$ lies in $L^{2}(0, T ; V)$ in view of (3.75), which allows the limit of (1.5) to hold as a relation in $L^{2}\left(0, T ; V^{\prime}\right)$ as specified by (2.25).

Moreover, recalling that $q \geq 2$ and using (3.85), (3.89), (3.90) and (3.94), we can easily check that

$$
\vartheta_{n}\left(\varphi_{n, t}+\boldsymbol{u}_{n} \cdot \nabla \varphi_{n}\right) \rightarrow \vartheta\left(\varphi_{t}+\boldsymbol{u} \cdot \nabla \varphi\right) \quad \text { weakly in } L^{p}((0, T) \times \Omega) \quad \text { for a suitable } p>1 \text {. }
$$

Next, thanks to (3.89) and (3.91), we get (actually, something more is true)

$$
\left|\nabla \boldsymbol{u}_{n}\right|^{2}+\left|\nabla \mu_{n}\right|^{2} \rightarrow|\nabla \boldsymbol{u}|^{2}+|\nabla \mu|^{2} \quad \text { strongly in } L^{1}((0, T) \times \Omega) .
$$

Relations (3.93)-(3.96) permit us to let $n \searrow \infty$ in all equations of the system (with (1.5) replaced by (2.25) in the limit, as noted above). In particular, the regularity properties (3.89)-(3.92) are a direct consequence of our argument. Finally, it is worth noting that the limit functions $\boldsymbol{u}, \varphi, \vartheta$ also satisfy the initial conditions (2.26). Indeed, they are continuous with respect to time with values in suitable Banach spaces (and the corresponding uniform estimates (3.89)-(3.92) hold for the approximating sequences). Hence, we may conclude that the limit quadruple $(\boldsymbol{u}, \varphi, \mu, \vartheta)$ solves our system in the sense of Theorem 2.1, as desired.

\section{Approximation and local existence}

In this section we give some highlights regarding a possible approximation of system (1.1)-(1.5) and provide a proof of local existence by means of a fixed point argument of Schauder type. In order to reduce the length of the exposition we leave most technical details to the reader, just limiting ourselves to outline the main steps of the procedure.

Regularized system. For (small) $\varepsilon \in(0,1)$ we consider the following regularized statement:

$$
\begin{aligned}
& \operatorname{div} \boldsymbol{u}=0, \\
& \boldsymbol{u}_{t}+\boldsymbol{u} \cdot \nabla \boldsymbol{u}+\nabla p=\Delta \boldsymbol{u}-\operatorname{div}(\nabla \varphi \otimes \nabla \varphi), \\
& \varphi_{t}+\boldsymbol{u} \cdot \nabla \varphi=\Delta \mu, \\
& \mu=-\varepsilon \Delta \varphi_{t}-\Delta \varphi+F_{\varepsilon}^{\prime}(\varphi)-\vartheta, \\
& \vartheta_{t}+\boldsymbol{u} \cdot \nabla \vartheta+\vartheta\left(\varphi_{t}+\boldsymbol{u} \cdot \nabla \varphi\right)-\operatorname{div}(\kappa(\vartheta) \nabla \vartheta)=|\nabla \boldsymbol{u}|^{2}+T_{\varepsilon}\left(|\nabla \mu|^{2}\right) .
\end{aligned}
$$


The above system differs from the original one due in view of the additional term $-\varepsilon \Delta \varphi_{t}$ in (4.3), which provides further parabolic regularity to $\varphi$, and of the truncation operator $T_{\varepsilon}$ in (4.5), where

$$
T_{\varepsilon}(v):=\min \left\{\varepsilon^{-1}, \max \left\{-\varepsilon^{-1}, v\right\}\right\}, \quad \text { for } v:(0, T) \times \Omega \rightarrow \mathbb{R},
$$

which yields boundedness of the last term on the right hand side of (4.5). Moreover $F_{\varepsilon}$ is a smooth regularization of $F$ such that $F_{\varepsilon}^{\prime}$ is Lipschitz continuous. We assume that $F_{\varepsilon}$ still enjoys the coercivity property (2.8). We also truncate the initial temperature in such a way that

$$
\vartheta_{0, \varepsilon} \in H^{1}(\Omega) \cap L^{\infty}(\Omega), \quad \varepsilon \leq \vartheta_{0, \varepsilon} \leq \varepsilon^{-1} \text { a.e. in } \Omega .
$$

Then, local existence for (4.1)-(4.5), complemented with the initial data $\boldsymbol{u}_{0}, \varphi_{0}$ and $\vartheta_{0, \varepsilon}$, and with periodic boundary conditions, is proved via a fixed point argument detailed below. This is essentially divided into three separate lemmas. At first, we fix $\vartheta$ and $\boldsymbol{u}$ in the Cahn-Hilliard system (4.3)-(4.4).

Lemma 4.1. Let $\varepsilon \in(0,1)$ and let $R>0$ be a number, depending on the initial data and on $\varepsilon$, such that

$$
\left\|\varphi_{0}\right\|_{H^{3}(\Omega)}+\left\|\boldsymbol{u}_{0}\right\|_{V}+\left\|\vartheta_{0, \varepsilon}\right\|_{H^{1}(\Omega)}+\left\|\vartheta_{0, \varepsilon}\right\|_{L^{\infty}(\Omega)} \leq R .
$$

Let also

$$
\bar{\vartheta} \in L^{2}(0, T ; V), \quad \overline{\boldsymbol{u}} \in L^{2}\left(0, T ; L^{4}(\Omega)\right), \quad \text { with }\|\bar{\vartheta}\|_{L^{2}(0, T ; V)}+\|\overline{\boldsymbol{u}}\|_{L^{2}\left(0, T ; L^{4}(\Omega)\right)} \leq R .
$$

Then there exist unique functions $\varphi$ and $\mu$ satisfying, a.e in $(0, T) \times \Omega$, the system

$$
\begin{aligned}
& \varphi_{t}+\overline{\boldsymbol{u}} \cdot \nabla \varphi=\Delta \mu, \\
& \mu=-\varepsilon \Delta \varphi_{t}-\Delta \varphi+F_{\varepsilon}^{\prime}(\varphi)-\bar{\vartheta},
\end{aligned}
$$

with the initial condition $\left.\varphi\right|_{t=0}=\varphi_{0}$. Moreover, the following regularity properties hold:

$$
\begin{aligned}
& \varphi \in H^{1}\left(0, T ; H^{3}(\Omega)\right), \\
& \mu \in L^{2}\left(0, T ; H^{2}(\Omega)\right), \\
& \|\varphi\|_{H^{1}\left(0, T ; H^{3}(\Omega)\right)}+\|\mu\|_{L^{2}\left(0, T ; H^{2}(\Omega)\right)} \leq Q_{1}(R, T) .
\end{aligned}
$$

Here and below, $Q_{i}:\left(\mathbb{R}^{+}\right)^{2} \rightarrow \mathbb{R}^{+}, i=1,2, \ldots$, are computable functions, increasingly monotone in each of their arguments, whose expression may additionally depend on $\varepsilon$.

Proof. We just give the highlights. Actually, once $\overline{\boldsymbol{u}}$ and $\bar{\vartheta}$ are assigned, (4.10)-(4.11) is a semilinear pseudo-parabolic system with Lipschitz nonlinearity. Hence, existence is standard. For example, it could be proved by relying on a Faedo-Galerkin scheme, or on a time-discretization argument. The a-priori estimates corresponding to the regularity conditions (4.12)-(4.13) are the following ones: first, one reproduces the energy estimate for the complete system by testing (4.10) by $\mu$ and (4.11) by $\varphi_{t}$. Noticing that

$$
\int_{\Omega} \mu \overline{\boldsymbol{u}} \cdot \nabla \varphi=-\int_{\Omega} \varphi \overline{\boldsymbol{u}} \cdot \nabla \mu \leq\|\varphi\|_{L^{4}(\Omega)}\|\overline{\boldsymbol{u}}\|_{L^{4}(\Omega)}\|\nabla \mu\| \leq \frac{1}{2}\|\nabla \mu\|^{2}+c\|\varphi\|_{V}^{2}\|\overline{\boldsymbol{u}}\|_{L^{4}(\Omega)}^{2},
$$

an estimate follows by using (4.9) and applying the Gronwall Lemma. With this estimate at disposal we can test (4.11) by $\Delta^{2} \varphi_{t}$. Thanks to the fact that $\mu, \bar{\vartheta} \in L^{2}(0, T ; V)$ due to the energy estimate and to (4.9), and using the Lipschitz continuity of $F_{\varepsilon}^{\prime}$, it is then not difficult to obtain (4.12).

Subsequently, noting that the left hand side of (4.10) lies in $L^{2}(0, T ; H)$, by elliptic regularity we obtain (4.13). Then, relation (4.14) is also a direct consequence of the a-priori estimates, as one can see by writing them in a quantitative way. Finally, uniqueness in the regularity class specified by (4.12)-(4.13) can be proved by a standard contractive argument. Namely, one may test (the difference of) (4.10) by $\varphi_{1}-\varphi_{2}$ (where $\left(\varphi_{1}, \mu_{1}\right)$ and $\left(\varphi_{2}, \mu_{2}\right)$ are two solutions) and (the difference of) (4.11) by $\Delta\left(\varphi_{1}-\varphi_{2}\right)$ and perform standard calculations. 
Lemma 4.2. Let us assume that the hypotheses of Lemma 4.1 are satisfied, and let $\varphi, \mu$ be the functions provided by Lemma 4.1. Then, there exists a unique function $\boldsymbol{u}$ such that

$$
\begin{aligned}
& \boldsymbol{u} \in H^{1}(0, T ; H) \cap L^{\infty}(0, T ; V) \cap L^{2}\left(0, T ; H^{2}(\Omega)\right), \\
& \|\boldsymbol{u}\|_{H^{1}(0, T ; H)}+\|\boldsymbol{u}\|_{L^{\infty}(0, T ; V)}+\|\boldsymbol{u}\|_{L^{2}\left(0, T ; H^{2}(\Omega)\right)} \leq Q_{2}(R, T) .
\end{aligned}
$$

Moreover, $\boldsymbol{u}$ satisfies, a.e in $(0, T) \times \Omega$, the system

$$
\begin{aligned}
& \operatorname{div} \boldsymbol{u}=0, \\
& \boldsymbol{u}_{t}+\boldsymbol{u} \cdot \nabla \boldsymbol{u}+\nabla p-\Delta \boldsymbol{u}=-\operatorname{div}(\nabla \varphi \otimes \nabla \varphi),
\end{aligned}
$$

with the initial condition $\left.\boldsymbol{u}\right|_{t=0}=\boldsymbol{u}_{0}$.

Proof. Also in this case we just give the highlights. Actually, as $\varphi$ is given satisfying (4.12) and (4.14), it is clear that the right hand side of (4.19) lies in $L^{2}(0, T ; H)$. Hence, existence and uniqueness of a solution satisfying (4.16) follow from the general theory of Navier-Stokes systems (cf., e.g., [31] or [36]). Moreover, writing explicitly the a-priori bounds, one also immediately gets (4.17), where, in principle, the expression of $Q_{2}$ may also depend on a suitable norm of $\varphi$. However, thanks to (4.14), $Q_{2}$ can in fact be written as a (computable and monotone) function of $R$ and $T$.

Finally, we come to the "heat" equation, which is a little bit more involved to deal with:

Lemma 4.3. Let the assumptions of Lemma 4.1 hold and let $\varphi, \mu, \boldsymbol{u}$ be the functions provided by Lemmas 4.1, 4.2. Then, there exists a unique function $\vartheta$ such that

$$
\begin{aligned}
& \vartheta \in H^{1}(0, T ; H) \cap L^{\infty}(0, T ; V) \cap L^{2}\left(0, T ; H^{2}(\Omega)\right) \cap L^{\infty}((0, T) \times \Omega), \quad \vartheta>0 \text { a.e. in }(0, T) \times \Omega, \\
& \|\vartheta\|_{H^{1}(0, T ; H)}+\|\vartheta\|_{L^{\infty}(0, T ; V)}+\|\vartheta\|_{L^{2}\left(0, T ; H^{2}(\Omega)\right)} \leq Q_{3}(R, T) .
\end{aligned}
$$

Moreover, $\vartheta$ satisfies, a.e in $(0, T) \times \Omega$,

$$
\vartheta_{t}+\boldsymbol{u} \cdot \nabla \vartheta+\vartheta\left(\varphi_{t}+\boldsymbol{u} \cdot \nabla \varphi\right)-\operatorname{div}(\kappa(\vartheta) \nabla \vartheta)=|\nabla \boldsymbol{u}|^{2}+T_{\varepsilon}\left(|\nabla \mu|^{2}\right),
$$

with the initial condition $\left.\vartheta\right|_{t=0}=\vartheta_{0, \varepsilon}$.

Proof. Equation (4.22) enjoys the quasilinear structure

$$
\vartheta_{t}+\boldsymbol{u} \cdot \nabla \vartheta+m_{1} \vartheta-\Delta K(\vartheta)=f
$$

where $K$ was defined in (2.12), and, in view of estimates (4.17), (4.14) and of interpolation, it is not difficult to infer

$$
\begin{aligned}
& f:=|\nabla \boldsymbol{u}|^{2}+T_{\varepsilon}\left(|\nabla \mu|^{2}\right) \in L^{p}((0, T) \times \Omega) \text { for all } p \in(1, \infty), \\
& m_{1}:=\varphi_{t}+\boldsymbol{u} \cdot \nabla \varphi \in L^{1}\left(0, T ; L^{\infty}(\Omega)\right) \cap L^{2}\left(0, T ; L^{p}(\Omega)\right) \text { for all } p \in(1, \infty) .
\end{aligned}
$$

Hence, existence of solutions to the initial-boundary value problem for (4.23) follows, as before, from standard techniques (as before, one could use time discretization or Faedo-Galerkin approximation).

Moreover, testing (4.23) by $\vartheta$ and performing simple calculations, we obtain the a-priori estimates leading to the regularity $\vartheta \in L^{\infty}(0, T ; H) \cap L^{2}(0, T ; V)$. By virtue of the high summability of $f$ and $m_{1}$, a standard application of Moser's iteration technique (see, e.g., [24, Chap. 5]) yields $\vartheta \in L^{\infty}((0, T) \times \Omega)$.

Strict positivity of $\vartheta$ follows from the maximum principle. Next, to deduce (4.20)-(4.21) one tests (4.22) by $K(\vartheta)_{t}$. Recalling (2.11), and using the $L^{\infty}$-bound for $\vartheta$ coming from Moser's iterations, we then get the estimate

$$
\begin{aligned}
& \int_{\Omega}\left(1+\vartheta^{q}\right)\left|\vartheta_{t}\right|^{2}+\frac{1}{2} \frac{\mathrm{d}}{\mathrm{d} t}\|\nabla K(\vartheta)\|^{2}=\int_{\Omega}\left(f-\boldsymbol{u} \cdot \nabla \vartheta-m_{1} \vartheta\right)\left(1+\vartheta^{q}\right) \vartheta_{t} \\
& \quad \leq \frac{1}{2}\left\|\vartheta_{t}\right\|^{2}+c\left\|f-\boldsymbol{u} \cdot \nabla \vartheta-m_{1} \vartheta\right\|^{2}\left(1+\|\vartheta\|_{L^{\infty}(\Omega)}^{2 q}\right) \\
& \quad \leq \frac{1}{2}\left\|\vartheta_{t}\right\|^{2}+c\|f\|^{2}+c\left(\|\boldsymbol{u}\|_{L^{\infty}(\Omega)}^{2}+\left\|m_{1}\right\|_{L^{4}(\Omega)}^{2}\right)\|\vartheta\|_{V}^{2} .
\end{aligned}
$$


Hence, noting that $\|\vartheta\|_{V} \leq c\|K(\vartheta)\|_{V}$ by $(2.11)$, conditions $\vartheta \in H^{1}(0, T ; H)$ and $K(\vartheta) \in L^{\infty}(0, T ; V)$ follow from Gronwall's lemma. Consequently we also have $\vartheta \in L^{\infty}(0, T ; V)$. In addition to that, viewing (4.23) as a timedependent family of elliptic problems (for the variable $K(\vartheta)$ ) with $L^{2}$-data and applying standard regularity results, we obtain that $K(\vartheta) \in L^{2}\left(0, T ; H^{2}(\Omega)\right)$.

Let now $k:=K(\vartheta)$ and denote as $\eta_{q}$ the inverse function of $K$ over $[0,+\infty)$. Observe that $\eta_{q}^{\prime}$ and $\eta_{q}^{\prime \prime}$ are uniformly bounded. Hence

$$
\Delta \vartheta=\Delta \eta_{q}(k)=\eta_{q}^{\prime}(k) \Delta k+\eta_{q}^{\prime \prime}(k)|\nabla k|^{2}
$$

belongs to $L^{2}(0, T ; H)$ thanks to the fact that $k \in L^{2}\left(0, T ; H^{2}(\Omega)\right) \cap L^{\infty}(0, T ; V)$. This entails (4.20). As before, as one writes explicitly the estimates leading to (4.20), also (4.21) follows.

Finally, to show uniqueness, we take two solutions $\vartheta_{1}$ and $\vartheta_{2}$ to (4.22) with the same initial datum and the same $\varphi, \mu, \boldsymbol{u}$. Then, the difference $\tilde{\vartheta}:=\vartheta_{1}-\vartheta_{2}$ solves

$$
\tilde{\vartheta}_{t}+\boldsymbol{u} \cdot \nabla \tilde{\vartheta}+m_{1} \tilde{\vartheta}-\Delta\left(K\left(\vartheta_{1}\right)-K\left(\vartheta_{2}\right)\right)=0 .
$$

Then, testing the above by sign $\tilde{\vartheta}$ (more precisely, one should first take an approximation of the sign function and then pass to the limit), using monotonicity of $K$, the incompressibility (4.18), and the periodic boundary conditions, we infer

$$
\frac{\mathrm{d}}{\mathrm{d} t}\|\tilde{\vartheta}\|_{L^{1}(\Omega)} \leq\left\|m_{1}\right\|_{L^{\infty}(\Omega)}\|\tilde{\vartheta}\|_{L^{1}(\Omega)}
$$

whence uniqueness follows from Gronwall's Lemma recalling the first condition in (4.25).

With the three lemmas at disposal, we can make explicit our fixed-point argument.

Theorem 4.4. Let $\varepsilon \in(0,1)$ and let us assume (4.6) and (4.7). Then there exist a time $T_{0}$ (depending on $\varepsilon$ and on the initial data) and at least one quadruple $(\boldsymbol{u}, \varphi, \mu, \vartheta)$ such that

$$
\begin{aligned}
& \boldsymbol{u} \in H^{1}\left(0, T_{0} ; H\right) \cap L^{\infty}\left(0, T_{0} ; V\right) \cap L^{2}\left(0, T_{0} ; H^{2}(\Omega)\right), \\
& \varphi \in H^{1}\left(0, T_{0} ; H^{3}(\Omega)\right), \\
& \mu \in L^{2}\left(0, T_{0} ; H^{2}(\Omega)\right), \\
& \vartheta \in H^{1}\left(0, T_{0} ; H\right) \cap L^{\infty}\left(0, T_{0} ; V\right) \cap L^{2}\left(0, T_{0} ; H^{2}(\Omega)\right) \cap L^{\infty}\left(\left(0, T_{0}\right) \times \Omega\right),
\end{aligned}
$$

with $\vartheta>0$ a.e. in $\left(0, T_{0}\right) \times \Omega$, satisfying system (4.1)-(4.5) a.e. in $\left(0, T_{0}\right) \times \Omega$ and complying with the initial conditions

$$
\left.\boldsymbol{u}\right|_{t=0}=\boldsymbol{u}_{0},\left.\quad \varphi\right|_{t=0}=\varphi_{0},\left.\quad \vartheta\right|_{t=0}=\vartheta_{0, \varepsilon} .
$$

Proof. Given $\varepsilon>0$, we truncate the initial temperature as specified in (4.7). Then, we choose $R>0$ correspondingly (cf. (4.8)). Hence, we can consider the closed ball (cf. (4.9))

$$
\mathcal{B}:=\left\{(\bar{\vartheta}, \bar{u}):\|\bar{\vartheta}\|_{L^{2}\left(0, T_{0} ; V\right)}+\|\overline{\boldsymbol{u}}\|_{L^{2}\left(0, T_{0} ; L^{4}(\Omega)\right)} \leq R\right\},
$$

where $T_{0} \in(0, T]$ will be chosen later on. Notice that the chosen radius $R$ depends only on the initial data (and on $\varepsilon$ by the truncation applied to $\vartheta_{0}$ ). Let us consider the fixed point map (also depending on $\varepsilon$, of course)

$$
\mathcal{T}: \mathcal{B} \rightarrow L^{2}\left(0, T_{0} ; V\right) \times L^{2}\left(0, T_{0} ; L^{4}(\Omega)\right), \quad \mathcal{T}:(\bar{\vartheta}, \bar{u}) \mapsto(\vartheta, \boldsymbol{u}) .
$$

We aim at applying the Schauder fixed point theorem to the above map, for a sufficiently small choice of the final time $T_{0}>0$. To this aim, we can observe the following:

(a) The map $\mathcal{T}$ is continuous: this follows from the fact that the fixed point equations (4.10)-(4.11), (4.19) and (4.22) only contain Lipschitz or locally Lipschitz nonlinearities. To give a formal proof (which is omitted for brevity), one could just put together (and refine a bit) the contractive arguments used to prove uniqueness in the three fixed point lemmas.

(b) The map $\mathcal{T}$ is compact: this follows immediately from (4.20), (4.16) and the Aubin-Lions lemma. Indeed, the output of the map $\mathcal{T}$ lies in a bounded set of a space which is compactly embedded into $L^{2}\left(0, T_{0} ; V\right) \times$ $L^{2}\left(0, T_{0} ; L^{4}(\Omega)\right)$. 
(c) The map $\mathcal{T}$ takes values into $\mathcal{B}$. Indeed, thanks to (4.21), (4.17), and the continuous embedding $V \subset L^{4}(\Omega)$, we get

$$
\|\boldsymbol{u}\|_{L^{\infty}\left(0, T_{0} ; L^{4}(\Omega)\right)}+\|\vartheta\|_{L^{\infty}\left(0, T_{0} ; V\right)} \leq Q_{4}\left(R, T_{0}\right),
$$

for some function $Q_{4}\left(R, T_{0}\right)$, whence

$$
\|\boldsymbol{u}\|_{L^{2}\left(0, T_{0} ; L^{4}(\Omega)\right)}+\|\vartheta\|_{L^{2}\left(0, T_{0} ; V\right)} \leq T_{0}^{1 / 2} Q_{4}\left(R, T_{0}\right) \leq R,
$$

provided $T_{0}$ is small enough.

In view of the above conditions (a)-(c) the assumptions of the Schauder fixed point argument are satisfied, whence (at least) one solution to (4.1)-(4.5) exists. The regularity conditions (4.30)-(4.33) follow immediately from the above three lemmas. Theorem 4.4 is proved.

We conclude this section with three observations aimed at clarifying the compatibility between the present approximation-fixed point argument and the a-priori estimates of the previous section.

Remark 4.5. In principle, the solution provided by the fixed point argument is local in time and the final time $T_{0}$ may depend on $\varepsilon$ and be smaller as smaller is $\varepsilon$. However, the a-priori estimates performed in the previous section are uniform with respect both to $\varepsilon$ and to time. This fact implies, first of all, that every approximate solution may be extended, at fixed $\varepsilon$, up to the original final time $T$ in the same regularity class specified by (4.30)-(4.33). Subsequently, we can take $\varepsilon \searrow 0$ working on the whole interval $(0, T)$ and obtain that also the solutions obtained as limit points of the approximating family have a global character.

Remark 4.6. It is also worth noting that neither the regularizing term $-\varepsilon \Delta \varphi_{t}$ added on the right hand side of (4.4) nor the truncation operator on the right hand side of (4.5) really interfer with the a-priori estimates of the previous section, which turn out to be independent of the approximation parameter $\varepsilon$. Actually, $-\varepsilon \Delta \varphi_{t}$ just gives some more information, vanishing as $\varepsilon$ goes to 0 , in the energy and subsequent bounds. On the other hand, the truncation operator yields some (positive) remainder term on the left hand side of the energy estimate, coming from the fact that, as one tests (4.3) by $\mu$ and (4.5) by 1 , the contribution of $\mu$ does not vanish completely. More precisely, the remaining non-negative terms on the left hand side of (3.2) take the form

$$
\int_{\Omega}\left(|\nabla \mu|^{2}-\varepsilon^{-1}\right)^{+}+\varepsilon \int_{\Omega}\left|\nabla \varphi_{t}\right|^{2} .
$$

Hence, it is immediate to see that the first term above compensates the fact that on the left hand side of the temperature estimate (3.14) only the truncation of $|\nabla \mu|^{2}$ would appear. Hence, (3.17) can still be obtained in the framework of our approximation.

Remark 4.7. Finally, we notice that, at least in principle, the regularity of approximating solutions, as specified in (4.30)-(4.33), is not sufficient in order for the estimates of the previous section to be fully rigorous. The main point stands in the fact that (4.32) does not give any information on the time derivative $\mu_{t}$, which would be needed as we perform the "Key estimate". However, it is easy to realize that (4.30)-(4.33) are just the outcome of the fixed point argument, and, hence, are not at all optimal. Indeed, further regularity of approximating solutions might be standardly proved by working separately on the equations of the approximate system and performing some bootstrap argument. This procedure may require a notable amount of technical work including a further regularization of the initial data. We decided to omit it since no effective mathematical difficulties seem to be involved. However, it is worth noting that, at least for what concerns the "heat" equation (which contains the most delicate nonlinearities), the regularity obtained in the fixed-point argument is sufficient. So, it would be enough to bootstrap regularity for $\mu$ and $\varphi$, which, as said, is a quite standard issue.

\section{Conflict of interest statement}

The authors declare that there is no conflict of interests in connection with this paper. 


\section{Acknowledgements}

We would like to thank the anonymous referee for his/her careful reading of the manuscript, which greatly improved the quality of the paper, and for suggesting us the shorter proof of Lemma A.1. Moreover, we thank Gianni Gilardi and Cristina Tarsi for the helpful discussions on some technical parts of the work.

\section{Appendix A}

We prove here the two-dimensional interpolation-embedding inequality used in the proof of existence. It is worth noting that the result is independent of the use of periodic boundary conditions and holds in any smooth bounded subset of $\mathbb{R}^{2}$. We will give two different proofs, the first using the so-called Yudovich' optimization trick, the second and more direct one, which was pointed to our attention by the anonymous referee, using the Trudinger-Moser inequality.

Lemma A.1. Let $\mathcal{O}$ be a smooth bounded domain in $\mathbb{R}^{2}$. Then, there exists $c>0$ depending only on $\mathcal{O}$ such that

$$
\|\xi\|_{H^{1}(\mathcal{O})^{\prime}} \leq c\left(1+\|\xi\|_{L^{1}(\mathcal{O})} \log ^{1 / 2}\left(e+\|\xi\|_{L^{2}(\mathcal{O})}\right)\right)
$$

for any $\xi \in L^{2}(\mathcal{O})$.

Proof. We start by recalling (see, e.g., [37, (17), p. 479]) that, for all $p \in[1, \infty)$,

$$
\|v\|_{L^{p}(\mathcal{O})} \leq c p^{1 / 2}\|v\|_{H^{1}(\mathcal{O})} \text { for all } v \in H^{1}(\mathcal{O}),
$$

where the constant $c>0$ can be taken independent of $p$. The above inequality makes precise the rate of explosion of the embedding constant of $H^{1}$ into $L^{p}$ as $p$ becomes large. As before, the value of $c$ can vary in the computations below; in any case, $c$ will always be intended to be independent of $p$.

Let us identify, as before, $L^{2}(\mathcal{O})$ with its dual in such a way that $L^{2}(\mathcal{O})$ can be (compactly and continuously) embedded into $H^{1}(\mathcal{O})^{\prime}$. Then, given $\eta \in L^{2}(\mathcal{O})$, we have, for $c>0$ as above,

$$
\frac{\langle\eta, v\rangle}{\|v\|_{H^{1}(\mathcal{O})}} \leq c p^{1 / 2} \frac{\langle\eta, v\rangle}{\|v\|_{L^{p}(\mathcal{O})}} \leq c p^{1 / 2}\|\eta\|_{L^{p^{\prime}(\mathcal{O})}},
$$

for all nonzero $v \in H^{1}(\mathcal{O})$ and where $p^{\prime}$ is the conjugate exponent to $p$. Hence,

$$
\|\eta\|_{H^{1}(\mathcal{O})^{\prime}} \leq c p^{1 / 2}\|\eta\|_{L^{p^{\prime}}(\mathcal{O})}=c p^{1 / 2}\|\eta\|_{L^{\frac{p}{p-1}(\mathcal{O})}} .
$$

Taking $p \geq 2$ and using interpolation, it then follows

$$
\|\eta\|_{H^{1}(\mathcal{O})^{\prime}} \leq c p^{1 / 2}\|\eta\|_{L^{1}(\mathcal{O})}^{\frac{p-2}{p}}\|\eta\|_{L^{2}(\mathcal{O})}^{\frac{2}{p}}
$$

Let us temporarily assume that $\|\eta\|_{L^{2}(\mathcal{O})}=1$. Then, squaring, we obtain

$$
\|\eta\|_{H^{1}(\mathcal{O})^{\prime}}^{2} \leq c p\|\eta\|_{L^{1}(\mathcal{O})}^{\frac{2(p-2)}{p}}=c p\|\eta\|_{L^{1}(\mathcal{O})}^{2}\|\eta\|_{L^{1}(\mathcal{O})}^{-\frac{4}{p}} .
$$

Now we use the so-called Yudovich' trick (see, e.g., [38]), namely we optimize the above right hand side with respect to $p \in[2, \infty)$. To this aim, let us consider the function

$$
f:[2, \infty) \rightarrow(0, \infty), \quad f(p):=p A^{-\frac{4}{p}},
$$

where $A>0$ is given. Then, clearly,

$$
f^{\prime}(p)=A^{-\frac{4}{p}}\left(1+\frac{4}{p} \log A\right) .
$$

Now, we have to distinguish between two cases. Firstly, if $A \geq e^{-1 / 2}$, then $f$ is increasing over $[2,+\infty)$, whence its minimum is achieved for $p=2$ :

$$
\min f=f(2)=2 A^{-2} \leq 2 e .
$$


On the other hand, if $A \in\left(0, e^{-1 / 2}\right)$, then $-4 \log A$ (the zero of $f^{\prime}$ ) is strictly larger than 2 , whence

$$
\min f=f(-4 \log A)=-4 A^{\frac{1}{\log A}} \log A=-4 e \log A .
$$

Let us now choose $A=\|\eta\|_{L^{1}(\mathcal{O})}$ for $\eta \in L^{2}(\mathcal{O})$ with $\|\eta\|_{L^{2}(\mathcal{O})}=1$. Then,

$$
\|\eta\|_{H^{1}(\mathcal{O})^{\prime}}^{2} \leq \begin{cases}2 e c\|\eta\|_{L^{1}(\mathcal{O})}^{2} & \text { if }\|\eta\|_{L^{1}(\mathcal{O})} \geq e^{-1 / 2} \\ -4 e c\|\eta\|_{L^{1}(\mathcal{O})}^{2} \log \left(\|\eta\|_{L^{1}(\mathcal{O})}\right) & \text { if }\|\eta\|_{L^{1}(\mathcal{O})}<e^{-1 / 2} .\end{cases}
$$

Let us now take any nonzero $\xi \in L^{2}(\mathcal{O})$ and apply the above to $\eta=\xi /\|\xi\|_{L^{2}(\mathcal{O})}$. If

$$
\|\eta\|_{L^{1}(\mathcal{O})} \geq e^{-1 / 2} \text {, i.e. }\|\xi\|_{L^{2}(\mathcal{O})} \leq e^{1 / 2}\|\xi\|_{L^{1}(\mathcal{O})},
$$

then it follows from the first (A.11) that

$$
\|\xi\|_{H^{1}(\mathcal{O})^{\prime}}^{2} \leq 2 e c\|\xi\|_{L^{1}(\mathcal{O})}^{2}
$$

and, in particular, (A.1) holds. On the other hand, if

$$
\|\eta\|_{L^{1}(\mathcal{O})}<e^{-1 / 2}, \quad \text { i.e. }\|\xi\|_{L^{2}(\mathcal{O})}>e^{1 / 2}\|\xi\|_{L^{1}(\mathcal{O})},
$$

then from the second (A.11) we obtain

$$
\|\xi\|_{H^{1}(\mathcal{O})^{\prime}}^{2} \leq 4 e c\|\xi\|_{L^{1}(\mathcal{O})}^{2}\left(\log \|\xi\|_{L^{2}(\mathcal{O})}-\log \|\xi\|_{L^{1}(\mathcal{O})}\right) .
$$

Here, we have to distinguish again some cases. First, if it is both $\|\xi\|_{L^{2}(\mathcal{O})} \geq 1$ and $\|\xi\|_{L^{1}(\mathcal{O})} \geq 1$, then (A.15) is continued as

$$
\|\xi\|_{H^{1}(\mathcal{O})^{\prime}}^{2} \leq 4 e c\|\xi\|_{L^{1}(\mathcal{O})}^{2} \log \|\xi\|_{L^{2}(\mathcal{O})}
$$

whence (A.1) follows. Second, if $\|\xi\|_{L^{2}(\mathcal{O})} \geq 1$ and $\|\xi\|_{L^{1}(\mathcal{O})}<1$, then, observing that $0 \leq-r \log r \leq c$ for all $r \in(0,1)$, we get

$$
\|\xi\|_{H^{1}(\mathcal{O})^{\prime}}^{2} \leq 4 e c\|\xi\|_{L^{1}(\mathcal{O})}^{2} \log \|\xi\|_{L^{2}(\mathcal{O})}-4 e c\|\xi\|_{L^{1}(\mathcal{O})}^{2} \log \|\xi\|_{L^{1}(\mathcal{O})} \leq 4 e c\|\xi\|_{L^{1}(\mathcal{O})}^{2} \log \|\xi\|_{L^{2}(\mathcal{O})}+c,
$$

and we still have (A.1). Finally, if it is both $\|\xi\|_{L^{2}(\mathcal{O})}<1$ and $\|\xi\|_{L^{1}(\mathcal{O})}<1$ then, we simply observe that $\|\xi\|_{H^{1}(\mathcal{O})^{\prime}}^{2} \leq$ $c\|\xi\|_{L^{2}(\mathcal{O})}^{2} \leq c$, which concludes the proof.

Proof. A more direct proof can be given starting from Trudinger-Moser's inequality. Namely, setting $\Phi(r):=$ $\exp \left(r^{2}\right)$, it is well-known that

$$
\sup _{\|\nabla z\|_{L^{2}(\mathcal{O})} \leq 1} \int_{\mathcal{O}} \Phi(z) \leq c(\mathcal{O})<\infty .
$$

Then, taking $v \in H^{1}(\mathcal{O})$ with $\|v\|_{H^{1}(\mathcal{O})} \leq 1$ and $\xi \in L^{2}(\mathcal{O}) \backslash\{0\}$, we have

$$
\begin{aligned}
\int_{\mathcal{O}} \xi v & \leq \int_{\mathcal{O}} \xi\left(v-v_{\mathcal{O}}\right)+v_{\mathcal{O}}\|\xi\|_{L^{1}(\mathcal{O})} \leq \int_{\mathcal{O}} \Phi\left(v-v_{\mathcal{O}}\right)+\int_{\mathcal{O}} \Phi^{*}(\xi)+c|\mathcal{O}|^{1 / 2}\|\xi\|_{L^{1}(\mathcal{O})} \\
& \leq c(\mathcal{O})+\int_{\mathcal{O}} \Phi^{*}(\xi)+c\|\xi\|_{L^{1}(\mathcal{O})} .
\end{aligned}
$$

Now, the convex conjugate function $\Phi^{*}$ cannot be computed directly. However, it is not difficult to check that

$$
\Phi^{*}(r) \leq|r| \log ^{1 / 2}(1+|r|) \quad \text { for all } r \in \mathbb{R} .
$$

Indeed, $\Phi^{*}$ is even and one may verify separately that $\Phi^{*}(r) \leq 0$ for $0 \leq r \leq 2$ and that $\left(\Phi^{*}\right)^{\prime}(r) \leq \log ^{1 / 2} r$ for $r \geq 1$. Thanks to (A.20), (A.19) gives

$$
\|\xi\|_{H^{1}(\mathcal{O})^{\prime}} \leq c\left(1+\|\xi\|_{L^{1}(\mathcal{O})}\right)+\int_{\mathcal{O}}|\xi| \log ^{1 / 2}(1+|\xi|) .
$$


Next, noting that the function $r \mapsto \log ^{1 / 2}(1+r)$ is concave and applying Jensen's inequality with respect to the measure $\mathrm{d} m:=\|\xi\|_{L^{1}(\mathcal{O})}^{-1} \mathrm{~d}|\xi|$, (A.21) can be continued as follows

$$
\begin{aligned}
\|\xi\|_{H^{1}(\mathcal{O})^{\prime}} & \leq c\left(1+\|\xi\|_{L^{1}(\mathcal{O})}\right)+\|\xi\|_{L^{1}(\mathcal{O})} \int_{\mathcal{O}} \log ^{1 / 2}(1+|\xi|) \mathrm{d} m \\
& \leq c\left(1+\|\xi\|_{L^{1}(\mathcal{O})}\right)+\|\xi\|_{L^{1}(\mathcal{O})} \log ^{1 / 2}\left(1+\int_{\mathcal{O}}|\xi| \mathrm{d} m\right) \\
& \leq c\left(1+\|\xi\|_{L^{1}(\mathcal{O})}\right)+\|\xi\|_{L^{1}(\mathcal{O})} \log ^{1 / 2}\left(1+\frac{\|\xi\|_{L^{2}(\mathcal{O})}^{2}}{\|\xi\|_{L^{1}(\mathcal{O})}}\right) \\
& \leq c\left(1+\|\xi\|_{L^{1}(\mathcal{O})} \log ^{1 / 2}\left(1+\|\xi\|_{L^{2}(\mathcal{O})}\right)\right)
\end{aligned}
$$

where the last inequality follows immediately by distinguishing between the cases $\|\xi\|_{L^{1}(\mathcal{O})} \leq 1$ and $\|\xi\|_{L^{1}(\mathcal{O})}>1$.

\section{References}

[1] H. Abels, On a diffuse interface model for two-phase flows of viscous, incompressible fluids with matched densities, Arch. Ration. Mech. Anal. 194 (2009) 463-506.

[2] H. Abels, Longtime behavior of solutions of a Navier-Stokes/Cahn-Hilliard system, in: Proceedings of the Conference "Nonlocal and Abstract Parabolic Equations and Their Applications", Bedlewo, in: Banach Cent. Publ., vol. 86, 2009, pp. 9-19.

[3] D.M. Anderson, G.B. MacFadden, A.A. Wheeler, Diffuse-interface methods in fluid mechanics, Annu. Rev. Fluid Mech. 30 (1998) $139-165$.

[4] F. Boyer, Mathematical study of multi-phase flow under shear through order parameter formulation, Asymptot. Anal. 20 (1999) 175-212.

[5] C. Cao, C.G. Gal, Global solutions for the 2D NS-CH model for a two-phase flow of viscous, incompressible fluids with mixed partial viscosity and mobility, Nonlinearity 25 (2012) 3211-3234.

[6] H. Brezis, Functional Analysis, Sobolev Spaces and Partial Differential Equations, Universitext, Springer, New York, 2011.

[7] F. Brezzi, G. Gilardi, FEM Mathematics, in: H. Kardestuncer (Ed.), Finite Element Handbook, McGraw-Hill Book Co., New York, 1987. Part I: Chapt. 1: Functional Analysis, pp. 1.1-1.5; Chapt. 2: Functional Spaces, pp. 2.1-2.11; Chapt. 3: Partial Differential Equations, pp. 3.1-3.6.

[8] M. Bulíček, E. Feireisl, J. Málek, A Navier-Stokes-Fourier system for incompressible fluids with temperature dependent material coefficients, Nonlinear Anal., Real World Appl. 10 (2009) 992-1015.

[9] J.W. Cahn, S.M. Allen, A microscopic theory for domain wall motion and its experimental verification in Fe-Al alloy domain growth kinetics, J. Phys. C 7 (38) (1977) 51-54.

[10] J. Cahn, J. Hilliard, Free energy of a nonuniform system. I. Interfacial free energy, J. Chem. Phys. 28 (1958) 258-267.

[11] M. Eleuteri, E. Rocca, G. Schimperna, On a non-isothermal diffuse interface model for two-phase flows of incompressible fluids, Discrete Contin. Dyn. Syst. 35 (2015) 2497-2522.

[12] E. Feireisl, Mathematical theory of compressible, viscous, and heat conducting fluids, Comput. Math. Appl. 53 (2007) 461-490.

[13] E. Feireisl, H. Petzeltová, E. Rocca, Existence of solutions to some models of phase changes with microscopic movements, Math. Methods Appl. Sci. 32 (2009) 1345-1369.

[14] M. Frémond, Non-smooth Thermomechanics, Springer-Verlag, Berlin, 2002.

[15] C.G. Gal, M. Grasselli, Asymptotic behavior of a Cahn-Hilliard-Navier-Stokes system in 2D, Ann. Inst. Henri Poincaré, Anal. Non Linéaire 27 (2010) 401-436.

[16] C.G. Gal, M. Grasselli, Trajectory attractors for binary fluid mixtures in 3D, Chin. Ann. Math., Ser. B 31 (2010) 655-678.

[17] C.G. Gal, M. Grasselli, Instability of two-phase flows: a lower bound on the dimension of the global attractor of the Cahn-Hilliard-NavierStokes system, Phys. D 240 (2011) 629-635.

[18] Z. Guo, P. Lin, A thermodynamically consistent phase-field model for two-phase flows with thermocapillary effects, arXiv:1401.5793v4, 2014.

[19] M.E. Gurtin, D. Polignone, J. Viñals, Two-phase binary fluids and immiscible fluids described by an order parameter, Math. Models Methods Appl. Sci. 6 (1996) 815-831.

[20] M. Heida, J. Málek, K.R. Rajagopal, On the development and generalizations of Cahn-Hilliard equations within a thermodynamic framework, Z. Angew. Math. Phys. 63 (2012) 145-169.

[21] P.C. Hohenberg, B.I. Halperin, Theory of dynamical critical phenomena, Rev. Mod. Phys. 49 (1977) 435-479.

[22] D. Jasnow, J. Viñals, Coarse-grained description of thermo-capillary flow, Phys. Fluids 8 (1996) 660-669.

[23] J.S. Kim, Phase-field models for multi-component fluid flows, Commun. Comput. Phys. 12 (2012) 613-661.

[24] O.A. Ladyzhenskaja, V.A. Solonnikov, N.N. Ural'ceva, Linear and Quasilinear Equations of Parabolic Type, Transl. Math. Monogr., vol. 23, American Mathematical Society, Providence, RI, 1968.

[25] A.G. Lamorgese, D. Molin, R. Mauri, Phase field approach to multiphase flow modeling, Milan J. Math. 79 (2011) $597-642$.

[26] J.L. Lions, Quelques Méthodes de Résolution des Problèmes aux Limites non Linéaires, Dunod Gauthier-Villars, Paris, 1969. 
[27] C. Liu, J. Shen, A phase field model for the mixture of two incompressible fluids and its approximation by a Fourier spectral method, Phys. D 179 (2003) 211-228.

[28] F. Luterotti, U. Stefanelli, Existence result for the one-dimensional full model of phase transitions, Z. Anal. Anwend. 21 (2002) 335-350.

[29] F. Luterotti, U. Stefanelli, Errata and addendum to "Existence result for the one-dimensional full model of phase transitions", Z. Anal. Anwend. 21 (2002) 335-350; Z. Anal. Anwend. 22 (2003) 239-240.

[30] A. Miranville, S. Zelik, Robust exponential attractors for Cahn-Hilliard type equations with singular potentials, Math. Methods Appl. Sci. 27 (2004) 545-582.

[31] J.C. Robinson, Infinite-Dimensional Dynamical Systems, Cambridge Texts Appl. Math., Cambridge University Press, 2001.

[32] E. Rocca, R. Rossi, "Entropic" solutions to a thermodynamically consistent PDE system for phase transitions and damage, SIAM J. Math. Anal. (2015), in press, arXiv:1403.2577v1,2014.

[33] J. Simon, Compact sets in the space $L^{p}(0, T ; B)$, Ann. Mat. Pura Appl. (4) 146 (1987) 65-96.

[34] V.N. Starovoitov, The dynamics of a two-component fluid in the presence of capillary forces, Math. Notes 62 (1997) $244-254$.

[35] P. Sun, C. Liu, J. Xu, Phase field model of thermo-induced Marangoni effects in the mixtures and its numerical simulations with mixed finite element method, Commun. Comput. Phys. 6 (2009) 1095-1117.

[36] R. Temam, Navier-Stokes Equations. Theory and Numerical Analysis, Stud. Math. Appl., vol. 2, North-Holland Publishing Co., Amsterdam, New York, Oxford, 1977.

[37] N.S. Trudinger, On imbeddings into Orlicz spaces and some applications, J. Math. Mech. 17 (1967) 473-483.

[38] V.I. Yudovich, Some estimates connected with integral operators and with solutions of elliptic equations, Dokl. Akad. Nauk SSSR 138 (1961) 805-808.

[39] Ya.B. Zel'dovich, Yu.P. Raizer, Physics of Shock Waves and High-Temperature Hydrodynamic Phenomena, Academic Press, New York, 1966.

[40] K. Zhao, Large time behavior of a Cahn-Hilliard-Boussinesq system on a bounded domain, Electron. J. Differ. Equ. 2011 (2011) 1-21.

[41] L. Zhao, H. Wu, H. Huang, Convergence to equilibrium for a phase-field model for the mixture of two viscous incompressible fluids, Commun. Math. Sci. 7 (2009) 939-962.

[42] Y. Zhou, J. Fan, The vanishing viscosity limit for a 2D Cahn-Hilliard-Navier-Stokes system with a slip boundary condition, Nonlinear Anal., Real World Appl. 14 (2013) 1130-1134. 